\title{
Perilipin 5 restores the formation of lipid droplets in activated hepatic stellate cells and inhibits their activation
}

\author{
Jianguo Lin and Anping Chen
}

Hepatic stellate cells (HSC) are major effectors during hepatic fibrogenesis. The activation of HSC is coupled to the loss of lipid droplets (LDs), which are specialized organelles composed of neutral lipids surrounded by perilipins. LDs have emerged as a focal point of interest in understanding the metabolic regulation of intrahepatic lipids during lipid-mediated liver fibrogenesis. Perilipin 5 (Plin5) is a newly identified LD protein in the perilipin family, which plays a key role in regulating aspects of intracellular trafficking, signaling, and cytoskeletal organization in hepatocytes. Recent work in Plin5 knockout mice suggests a role in high fat diet-induced hepatic lipotoxicity. The current report is to evaluate the impact of Plin5 on HSC activation and to elucidate the underlying mechanisms. We now show that high fat diet-induced liver fibrosis is accompanied by an approximate $75 \%$ reduction in Plin5 in HSC, and that spontaneous activation of primary HSC produces temporally coincident loss of Plin5 expression and LD depletion. As modulating lipid content in HSC is a suggested strategy for inhibition of HSC activation and treatment of hepatic fibrosis, we asked whether exogenous Plin5 expression in primary HSC would reverse the activation phenotype and promote LD formation. Recombinant lentiviral Plin5 expression in primary mouse HSC restored the formation of LDs, increased lipid content by inducing expression of pro-lipogenic genes and suppressing expression of pro-lipolytic genes, and suppressed HSC activation ( two fold reduction in expression of procollagen and $a$-smooth muscle actin, two unique biomarkers for activated HSC). In addition, the expression of exogenous Plin5 in HSC attenuated cellular oxidative stress by reducing cellular reactive oxygen species, elevating cellular glutathione, and inducing gene expression of glutamate-cysteine ligase. Taken together, our results indicate that expression of Plin5 plays a critical role in the formation of LDs, the elevation of lipid content in HSC, and the inhibition of the activation of HSC.

Laboratory Investigation (2016) 96, 791-806; doi:10.1038/labinvest.2016.53; published online 2 May 2016

Lipid droplets (LDs) exist in virtually all normal cells and consist of a triacylglycerol core with a phospholipid monolayer on the surface in which amphiphilic proteins insert. Proteins that coat LDs, ie, perilipins (Plins), have diverse functions that are not well elucidated in many tissues. Perilipins are crucial for the formation of cellular LDs and modulation of lipid homeostasis by regulating the storage and utilization of intracellular lipids. ${ }^{1}$ Perilipins are numbered in the order of discovery. ${ }^{2}$ Plin1 (Perilipin) and Plin2 (Adipophilin) are exclusively associated with LDs and are degraded when not bound to LDs. In contrast, Plin3 (TIP47) and Plin4 (S3-12) exchange on and off LDs and are stable in LDs or in the cytoplasm. ${ }^{3}$ Perilipin 5 (Plin5) (ie, myocardial LD protein) is a newly discovered member in the Plin family. 4,5
Plin5 is mainly expressed in highly oxidative tissues, such as heart, skeletal muscle, and liver. ${ }^{4,5}$ Recent work in Plin5 knockout mice suggests a role in high fat diet (HFD)-induced hepatic lipotoxicity. ${ }^{6}$

Hepatic fibrosis refers to the deposition of high-density extracellular matrix (ECM) protein forming scarring tissue due to an imbalance between fibrogenesis and fibrolysis. Liver fibrosis and in particular cirrhosis have become major endpoints in clinical trials of patients with chronic liver diseases. Viral hepatitis, alcoholic, and non-alcoholic steatohepatitis have become the major etiologies. ${ }^{7,8}$ Hepatic fibrosis is currently the target of significant scientific and clinical interest in hepatology. Very few breakthroughs have, however, occurred in therapeutic intervention of this disease. ${ }^{9}$ 
Research developing novel, safe, and effective anti-fibrotic strategies is, thus, of the highest priority. ${ }^{10}$

Hepatic stellate cells (HSC) are the major effectors during hepatic fibrogenesis. Quiescent HSC are featured by lipids within a large number of LDs in cytosol. ${ }^{7,8,11}$ During liver fibrogenesis, an apparent change in activated HSC is loss of LDs and depletion of cellular lipids, in addition to enhanced cell proliferation, de novo expression of $\alpha$-smooth muscle actin $(\alpha$-SMA), and over-production of extracellular matrix. ${ }^{7,8,11}$ It has been shown that after 7 days of culture, quiescent HSC spontaneously become fully activated, ${ }^{11}$ mimicking the process seen in vivo, which provides a good and simple model for elucidating underlying molecular mechanisms of HSC activation and studying potential therapeutic intervention of the process. Most evolving antifibrotic therapies are aimed at inhibiting the activation of HSC.

Lipids present in HSC include retinyl ester, triglyceride (TG), cholesteryl ester, cholesterol, phospholipids, and free fatty acids (FFA). Retinyl ester and TG are present at similar concentrations. These two classes of lipids account for approximately three-quarters of the total lipids in LDs of HSC. ${ }^{1}$ Accumulating evidence has shown the importance of cellular lipids in maintaining HSC in quiescent state. ${ }^{12,13}$ Restoration of lipogenesis and elevation of cellular lipid content in HSC might be a strategy for attenuating HSC activation and inhibiting fibrogenesis. ${ }^{12,13}$

Our pilot experiments revealed that Plin 1 and Plin4 were hardly detectable in quiescent HSC or activated HSC (data not shown here). The level of Plin3 was low and not changed in HSC on activation. The level of Plin2 was dramatically reduced in activated HSC, which was consistent with prior observations. ${ }^{14}$ Expression and roles of Plin5 in the activation of HSC and in hepatic fibrogenesis remain largely unknown.

Although underlying mechanisms remain incompletely understood, accumulating evidence has indicated that oxidative stress plays a critical role in stimulating HSC activation. ${ }^{7,8,11}$ Oxidative stress is a deleterious imbalance between the production and the removal of free radicals, including reactive oxygen species (ROS). Reducing oxidative stress in mammalian cells is through several antioxidant systems, including enzymes and nonenzymatic molecules. Among them, glutathione (GSH) is the main non-protein thiol. It reacts with ROS or functions as a cofactor of antioxidant enzymes. GSH is converted to its oxidized form (GSSG), leading to the conversion of $\mathrm{H}_{2} \mathrm{O}_{2}$ and lipid peroxides to water and lipid alcohols catalyzed by GSH peroxidase. This process thereby prevents degradation to highly toxic free radicals. GSSG is ultimately reduced to GSH by GSH reductase to maintain cellular GSH at a proper level. The ratio of GSH/GSSG is regarded as a sensitive indicator of oxidant stress. ${ }^{15,16}$ A higher ratio of GSH/GSSG indicates a lower level of oxidant stress. De novo synthesis of GSH requires glutamate-cysteine ligase (GCL), a key rate-limiting enzyme in GSH synthesis. ${ }^{17}$ The GCL enzyme is a heterodimer with a large catalytic subunit (GCLc, $\sim 73 \mathrm{kDa}$ ) and a small modifier subunit (GCLm, $\sim 30 \mathrm{kDa})$, which are encoded by different genes and dissociated under reducing conditions. ${ }^{18,19}$ LDs prevent excess production of ROS by sequestering fatty acids from oxidation and hence suppress oxidative stress. ${ }^{20}$

The aim of this study is aimed at elucidating the role of Plin5 in inhibiting HSC activation and exploring underlying mechanisms, we hypothesize that on activation during liver injury, the loss of LDs and the depletion of cellular lipids in HSC are coupled to an apparent reduction in gene expression of Plin5. The expression of exogenous Plin5 could restore the formation of LDs and elevate cellular lipid content in HSC, leading to inhibition of the activation of HSC.

\section{MATERIALS AND METHODS Materials}

Compound C (Compd C), a selective inhibitor for 5'-AMPactivated protein kinase (AMPK); and 5-aminoimidazole-4carboxamide-1- $\beta$-D-ribofuranoside (AICAR), an AMPK activator, were purchased from Sigma (St Louis, MO, USA). Antibodies used in this study were previously discussed, ${ }^{21-23}$ and purchased from Santa Cruz Biotechnology (Santa Cruz, CA, USA), except specific indications.

\section{Animal Studies}

The animal protocol for the use of mice in this study was approved by Institutional Animal Care and Use Committee of Saint Louis University. C57BL/6 J mice (Jackson Laboratory, Bar Harbor, MA, USA) were housed in a temperaturecontrolled animal facility $\left(23^{\circ} \mathrm{C}\right)$ with a $12: 12 \mathrm{~h}$ light-dark cycle and allowed free access to regular chow and water ad libitum, and were used in all in vivo studies. To generate an obese mouse model with hepatic fibrosis, C57BL/6 female mice $(n=20)$ were randomly divided into two groups. Hepatic steatosis with fibrosis in one group of mice $(n=10)$ was induced by feeding a high trans-fat diet supplemented with high fructose corn syrup for 16 weeks as described, ${ }^{24}$ with minor modifications, including drinking water in bottles and without removal of cage racks. ${ }^{25}$ The control group $(n=10)$ were fed a chow diet. After sacrifice, small pieces of different lobes from each mouse of the obese mouse model were collected for immuno-histochemical studies by fixation with formalin (10\%) and subsequent embedment with paraffin.

\section{Isolation and Culture of HSC}

HSC were isolated by the pronase-collagenase perfusion in situ before density gradient centrifugation, as we previously described. ${ }^{21}$ Freshly isolated HSC were used for RNA preparation, or were cultured in Dulbecco's modified Eagle's medium supplemented with $20 \%$ of fetal bovine serum. Cells were passaged in Dulbecco's modified Eagle's medium with $10 \%$ of fetal bovine serum. If no specific indication, semi- 
confluent HSC with four to nine passages were used in experiments.

\section{Western Blotting Analyses}

Preparation of whole-cell extracts, SDS-PAGE, transblotting, and subsequent immuno-reactions were conducted, as we previously described. ${ }^{21,23} \beta$-actin or $\beta$-tubulin was used as an invariant control for equal loading.

\section{RNA Extraction and Real-Time PCR}

Total RNA was extracted from cells by the TRI-Reagent, following the protocol recommended by the manufacturer (Sigma, St Louis, MO, USA). Total RNA was treated with DNase I before the synthesis of the first strand of cDNA. Realtime PCR were performed, as we previously described using SYBR Green Supermix. ${ }^{26}$ mRNA levels are expressed as fold changes after normalization with glyceraldehyde-3-phosphate dehydrogenase, as described by Schmittgen et al. ${ }^{27}$ The following primers were used in real-time PCR:

abdh5: (F) 5'-TCTTGCTTGGACACAACCTG-3', (R) 5'-G AGGTGACTAACCCTTGATGG-3';

ACC: (F) 5'-ACCAGCCTCTGTCAGCTCAG-3', (R) 5'-CC CCCAAAACGAGTAACAAA-3';

ATGL: (F) 5'-AACACCAGCATCCAGTTCAA-3', (R) 5'-G GTTCAGTAGGCCATTCCTC-3';

$\alpha 1$ (I)collagen: (F) 5'-TTCACCTACAGCACGCTTGTG-3', (R) 5'-GATGACTGTCTTGCCCCAAGTT-3';

FAS: (F) 5'-CTGTTGGAAGTCAGCTATGAAG-3',

5'-AGCTGGAGGAGCAGGCTGTG-3;

GCLc: (F) 5'-ATGACTGTTGCCAGGTGGATGAGA-3', (R)

5'-ACACGCCATCCTAAACAGCGATCA-3';

GCLm: (F) 5'-AGCTGGACTCTGTGATCATGGCTT-3', (R) 5'-CAAAGGCAGTCAAATCTGGTGGCA-3';

HSL: (F) 5'-ACGGATACCGATGTTTGGTGC-3' ${ }^{\prime}$, (R) 5' ${ }^{\prime}$-T CCAGAAGTGCACATCCAGGT-3';

LCPT: (F) 5'-CGGCTGCACTCCTGGAAGAAGAA- $3^{\prime}$, (R)

5'-CTCGGCCCCGCAGGTAGATGTA-3';

PGC-1 $\alpha$ : (F) 5'-ACACCTGTGACGCTTTCGCTG-3'，(R)

5'-CATTTGAAGGGGTCGCCCTTG-3';

Plin5: (F) 5'-CCATATGGACCAGAGAGGTGAAGACAC CAC-3', (R) 5'-GGAAGATCTTCAGGAGTCCAGCTCTG GCA-3';

PPAR- $\alpha$ : (F) $5^{\prime}$-AGGCTGTAAGGGCTTCTTTC-3'， (R) 5'-CGAATTGCATTGTGTGACAT-3';

PPAR- $\gamma:$ (F) 5'-ATGCCAAAAATATCCCTGGTTTC-3', (R) 5'-GGAGGCCAGCATGGTGTAGA-3';

$\alpha$-SMA: (F) 5'-TCAGCGCCTCCAGTTCCT-3', (R) 5'-AA AAAAACCACGAGTAACAAATCAA-3';

GAPDH: (F) 5'-CTACACTGAGGACCAGGTTGTCT-3', (R) 5'-GGTCTGGGATGGAAATTGTG-3'.

\section{Cell Proliferation Assays}

Cell growth was colorimetrically determined by using the non-radioactive cell proliferation assay kit (ie, MTS assays; Promega, Madison, WI, USA), following the protocol provided by the manufacturer. Each group was carried out in triplicates and repeated for at least three times. Results were expressed as percentage changes in the density of viable cells. $^{28}$

\section{Plasmids, Transient Transfection, and Luciferase Activity Assays}

The plasmid pPPRE-Luc was a luciferase activity reporter. It contained three copies of peroxisome proliferator response elements (PPRE) from the acyl-CoA oxidase gene. ${ }^{29}$ It was a gift from Dr Kevin J. McCarthy (Louisiana State University Health Sciences Center in Shreveport). The plasmid LXR-Luc was a luciferase activity reporter with three copies of liver X receptor (LXR) binding elements in the promoter region of the luciferase reporter plasmid pGL3. ${ }^{30}$ It was a gift from Dr Knut Steffensen (Department of Biosciences, Karolinska Institutet at NOVUM, Huddinge, Sweden). The plasmid TOPflash was a Wnt $/ \beta$-catenin signaling luciferase activity reporter. ${ }^{31}$ TOPflash contained eight copies of TCF-LEF binding sites in the promoter. It was kindly provided by Dr Randall Moon (Department of Pharmacology, School of Medicine, University of Washington, Seattle, WA, USA). The luciferase activity report plasmid p8xARE was a gift from Dr Roland Wolf, ${ }^{32}$ which contained eight copies of the transcription factor Nrf2 binding sites in the promoter. Both of the plasmids paAMPK (aAMPK) and pdn-AMPK (dn-AMPK) contained a full length of AMPK cDNA. paAMPK expressed constitutively activated AMPK, whereas pdn-AMPK expressed dominant-negative AMPK. Both plasmids were gifts from Dr David Carling. ${ }^{33}$ Plin5-Luc was generated, as we previously described. ${ }^{26}$ In brief, a $5^{\prime}$-fragment $(+2462 / 26 \mathrm{bp})$ of mouse Plin 5 promoter (NCBI Reference Sequence: NC_000083.6) was generated by PCR using primers: (F) 5'-GCTAGGTACC(KpnI) GCAGGAGTG TGTTCCAGCTGT-3', (R) 5'-GACTGCTAGC(NheI) GTGG TGTCTTCACCTCTCTG-3'. After digestion with KpnI and NheI, the cDNA fragment was subcloned into the luciferase activity reporter plasmid pGL3-Basic.

For co-transfection, semi-confluent HSC in six-well cell-culture plates were transiently transfected with a total of $4.5 \mu \mathrm{g}$ DNA per well, using the LipofectAMINE reagent (Invitrogen, Carlsbad, CA), as we previously described. ${ }^{26}$ It included $2 \mu \mathrm{g}$ of pPlin5-Luc, $0.5 \mu \mathrm{g}$ of pSV- $\beta$-gal, and $2.0 \mu \mathrm{g}$ of paAMPK, or pdn-AMPK, at various doses plus the empty vector pcDNA3. The latter was used to ensure an equal amount of total DNA in transfection assays. Each sample was in triplicate in every experiment. Transfection efficiency was normalized by co-transfection of the $\beta$-galactosidase reporter plasmid pSV- $\beta$-gal $(0.5 \mu \mathrm{g}$ per well) (Promega Corporation, Madison, WI, USA). $\beta$-galactosidase activities were measured by using a chemiluminescence assay kit (Tropix, Bedford, MA, USA). Luciferase activities were expressed as relative units after normalization with $\beta$-galactosidase activities per $\mathrm{mg}$ protein. Results were combined from at least three independent experiments. 


\section{Construction of the Recombinant Lentiviral LV-Plin5-YFP with a Full Length or Frangments of Plin5}

Total RNA was extracted from mouse liver tissues by using TRI reagent (Sigma). Mouse Plin5 cDNA was generated by PCR. The primers from NCBI Reference Sequence (NM_025874.3) were the followings:

A full length of Plin5 (aa1-463):

(F): 5'-TTGCGAGAATTC(EcoRI) ATGGACCAGAGAGG TGAAGACA-3' and (R): 5'-GTGGCGACCGGT(AgeI) GC GAAGTCCAGCTCTGGCATCATTG-3'.

Plin5 (aa1-188):

(F) 5'-TTGCGAGAATTCATGGACCAGAGAGGTGAAGA CACCAC-3'

(R) 5'-GTGGCGACCGGTGCTAGCTCAGCCTCAGTCAT GGG-3'

Plin5 (aa1-116):

(F) 5'-TTGCGAGAATTCATGGACCAGAGAGGTGAAGA CACCAC-3'

(R) 5'-GTGGCGACCGGTTTCTAATTTGTCTAGTCCCC TGCA-3'

Plin5 (aa117-188):

(F) 5'-TTGCGAGAATTCGAGAAGCTGCCCTTCCTGCAG-3'

(R) 5'-GTGGCGACCGGTGCTAGCTCAGCCTCAGTCAT GGG-3'

Plin5 (aa189-463):

(F) 5'-TTGCGAGAATTCATGGCAGTCCTGGCAGCTGA GGC-3'

(R) 5'-GTGGCGACCGGTGCGAAGTCCAGCTCTGGCA TCATTG-3'

The underlined six nucleotides were, respectively for generating an EcoRI restriction site at the $5^{\prime}$-end and an AgeI site at the $3^{\prime}$-end in the PCR products. cDNA was reversely transcribed using High Capacity RNA-to-cDNA kit (Applied Biosystems, CA, USA). After digestion with EcoRI and AgeI, the PCR products were subcloned into the lentiviral plasmid pFLRu-YFP digested with EcoRI and AgeI. ${ }^{34,35}$ The products were respectively called pFLRu-Plin5-YFP or pFLRu-Plin5YFP fragments.

To generate lentiviruses expressing Plin5 tagged with yellow fluorescent protein (YFP), sub-confluent Lenti-X $293 \mathrm{~T}$ cells, a lentiviral packaging cell line (Clontech Laboratories, Mountain View, CA) were co-transfected with pFLRu-Plin5-YFP or the control plasmid pFLRu-YFP, and a mixture (3:1) of lentiviral packaging plasmids $\mathrm{pHR} 8.2 \Delta \mathrm{R}$ and the envelop plasmid pCMV-VSVG. Media were refreshed for virus production $24 \mathrm{~h}$ after transfection. Media containing lentiviral particles, ie, the recombinant lentiviral LV-Plin5YFP or the vehicle lentiviral LV-YFP, were collected and filtered with syringe filters $(0.45 \mathrm{~mm})$ in the following day.

To generate transducted cells, cultured-mouse HSC at $70 \%$ confluence were transducted by adding LV-plin5-YFP or LV-YFP. Cells were transducted again in the same way $24 \mathrm{~h}$ after the first transduction. Forty-eight hours after the first transduction, positively transducted HSC were selected by puromycin $(5 \mu \mathrm{g} / \mathrm{ml})$ for additional $48 \mathrm{~h}$. Transducted cells showed fluorescence under a fluorescent microscope. Transducted HSC grew in media with puromycin at $2 \mu \mathrm{g} / \mathrm{ml}$ until proper population.

\section{Hematoxylin and Eosin Stain}

It was conducted as we previously described. ${ }^{21}$ Representative views were presented.

\section{Sirius Red Stain}

Mouse liver sections were deparaffinized with xylenes and dehydrated with ethanol. After rinsing with $\mathrm{ddH}_{2} \mathrm{O}$, the sections were stained with Weigert's iron hematoxylin solution. After rinsing, the sections were stained with Sirius Red/Fast Green solution for at least $2 \mathrm{~h}$ with mild agitation. Section slides were gently rinsed with $\mathrm{ddH}_{2} \mathrm{O}$. After dehydrated with ethanol and treated with xylenes, the sections were mounted in Permount. Representative views were presented.

\section{Immuno-Histochemistry}

$\alpha$-SMA in mouse livers was detected by immunehistochemistry using Histostain-Plus 3rd Gen IHC Detection Kit from Invitrogen (cat\# 85-9673, Invitrogen, CA, USA). Representative views were presented.

\section{Immuno-Fluorescent Stain}

The experiments were conducted as we previously described, ${ }^{21}$ using goat polyclonal primary antibody against Plin5 (1:50, sc-240627, Santa Cruz, CA, USA) and donkey anti-goat IgG secondary antibody conjugated with fluorescent Texas Red dye (1:300, cat\#705-075-147, Jackson ImmunoResearch, PA, USA). Nuclei were stained by a mounting solution with DAPI. The slides were observed under a fluorescent microscope (Leica DM 4000 B). Representative views of were presented.

\section{Oil Red O Staining}

HSC were seeded on autoclaved cover slips in a 6-well plate and cultured in Dulbecco's modified Eagle's medium with $10 \%$ fetal bovine serum for indicated days before fixation with $4 \%$ paraformaldehyde (30 min). LDs were stained, as we previously described. ${ }^{36}$

\section{Cellular ROS Assays}

Levels of ROS in HSC were determined by analyzing dichlorofluorescein fluorescence, as we previously described. ${ }^{37}$

\section{Lipid Peroxidation Assays (LPO)}

LPO assays were performed by using the Lipid Hydroperoxide Assay Kit purchased from Cayman Chemical, as we previously described. ${ }^{37}$ 


\section{Glutathione Assays}

Levels of reduced GSH and oxidized glutathione (GSSG) were determined by using the enzyme immune assay kit GSH-400 (Cayman Chemical), as we previously described. ${ }^{37}$

\section{Statistical Analyses}

Differences between means were evaluated using an unpaired two-sided Student's $t$-test $(P<0.05$ considered as significant $)$. Where appropriate, comparisons of multiple treatment conditions with controls were analyzed by ANOVA with the Dunnett's test for post hoc analysis.

\section{RESULTS}

\section{Plin5 is Highly Expressed in Quiescent HSC and} Dramatically Reduced in HSC on Activation In Vitro and In Vivo

To evaluate plin5 expression in HSC in vivo, an obese mouse model with hepatic fibrosis was generated, as we described. ${ }^{25}$ Mice fed a HFD were previously characterized and hepatic fibrosis in the mice was evaluated by real-time PCR analyses of pro-fibrogenic genes and quantitation of picro-Sirius Red and trichrome staining of collagens in liver tissue. ${ }^{25}$ As shown in Figure 1a, compared to mice in the chow group, mice fed HFD showed apparent deposition of extracellular matrix in the liver demonstrated by Sirius Red stain, indicating hepatic fibrosis in the group of HFD mice. Immuno-histochemical studies showed positive stain of $\alpha$-SMA, a unique marker for activated HSC, in the liver in the group of HFD mice. These results indicated, as expected, that mice fed HFD had hepatic fibrosis and many HSC, if not all, were activated. Total RNA were prepared from freshly isolated HSC from the HFD mice for real-time PCR assays. As shown in Figure 1b, compared with that in HSC from the chow group (Chow), the mRNA level of $\alpha 1$ (I) procollagen was, as expected, dramatically higher by $80 \%$ in HSC from the HFD group (HFD). Further experiments indicated that compared with that in HSC from the chow group, the mRNA level of Plin5 was significantly reduced by $\sim 75 \%$ in HSC from the HFD group. These results indicated that plin 5 expression was dramatically reduced in activated HSC in vivo.

It has been shown that after 7 days of culture, quiescent HSC spontaneously become fully activated. ${ }^{11}$ To further evaluate the expression of Plin5 in activated HSC, HSC from normal C57BL/6 mice were respectively cultured for $24 \mathrm{~h}$ (Day 1) and 7 days (Day 7). Total RNA was prepared for determining mRNA levels of $\alpha \mathrm{I}(\mathrm{I})$ procollagen (procol), $\alpha$-SMA and Plin 5 by real-time PCR. As shown in Figure 1c, compared with those in HSC after 1 day of culture (Day 1), mRNA levels of $\alpha \mathrm{I}(\mathrm{I})$ procollagen and $\alpha$-SMA were significantly elevated, as expected, in cells after 7 days of culture (Day 7). In great contrast, the mRNA level of Plin5 in cells of Day 7 was significantly reduced by $\sim 90 \%$. Immuno-stain of Plin5 in Figure 1d indicated that Plin5 was apparently observed in HSC at Day 1. However, Plin5 was hardly detectable in HSC at Day 7. Taken together, our results indicated that Plin5 was highly expressed in quiescent HSC and dramatically decreased in HSC on activation in vitro and in vivo. These results also suggest that the expression of Plin5 in HSC might facilitate the maintenance of HSC in quiescent state.

\section{The Expression of Exogenous Plin5 Inhibits HSC Activation}

To elucidate the effect of Plin5 on HSC activation, cultureactivated HSC (4-9 passages in culture) were respectively transducted with the recombinant lentiviral LV-Plin5-YFP, or the vehicle lentiviral LV-YFP, or no transduction as a mock control (Mock HSC). After selection of positively transducted cells with puromycin, total RNA or whole-cell extracts were prepared. As shown (Figures $2 \mathrm{a}$ and $\mathrm{b}$ ) by real-time PCR assays and western blotting analyses, as expected, Plin 5 was hardly detectable in mock HSC or HSC transducted with LVYFP. In great contrast, HSC transducted with LV-Plin5-YFP showed high levels of Plin5 demonstrated by real-time PCR and western blotting analyses. These data indicated that exogenous Plin5 could be introduced and expressed in activated HSC in vitro.

We assumed that expression of Plin5 could inhibit the activation of HSC. To test the assumption, passaged HSC were transducted with LV-Plin5-YFP, or LV-YFP, or no transduction. Cell growth was analyzed by MTS assays. As shown in Figure 2c, compared with mock HSC, cells transducted with LV-YFP showed no apparent change in cell growth. However, HSC with LV-Plin5-YFP showed a significant reduction in cell growth by $28 \%$. To further confirm the inhibitory impact of Plin5 on HSC activation, real-time PCR and western blotting analyses were conducted. As shown in Figures $2 \mathrm{~d}$ and e, compared with mock HSC, cells transducted with LV-Plin5-YFP showed a dramatic reduction in type I collagen and $\alpha$-SMA, two unique biomarkers for activated HSC, and a significant increase in PPAR $\gamma$, at levels of mRNA and protein. However, cells with LV-YFP had no such changes. Western blotting analyses confirmed the observations and further revealed that the expression of exogenous Plin5 dramatically reduced the levels of pro-fibrogenic transforming growth factor-beta receptor I and II (TGF $\beta$-RI and TGF $\beta$-RII). These results collectively demonstrated that the expression of exogenous Plin5 inhibited HSC activation in vitro.

\section{Exogenous Plin5 Restores the Formation of LD and Elevates Cellular Lipid Content in Activated HSC}

To elucidate the mechanisms by which exogenous Plin5 inhibited HSC activation, we postulated that expression of Plin5 stimulated the formation of LDs and elevated cellular lipid content in HSC, leading to the inhibition of HSC activation. To test the postulation, passaged HSC were transducted with LV-Plin5-YFP, or LV-YFP, or no transduction (Mock HSC). After selection with puromycin and culture 
a
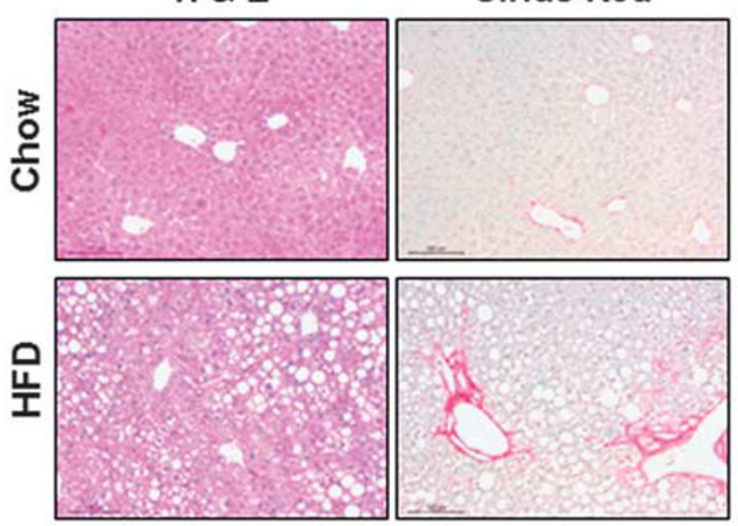

anti- $\alpha-S M A$
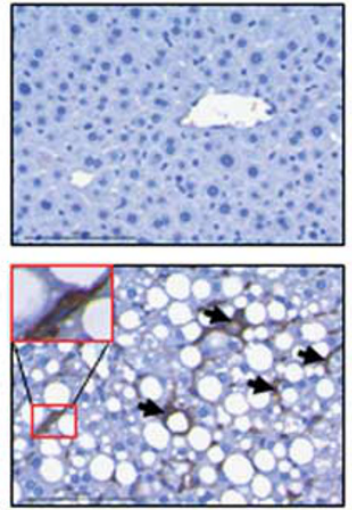

b

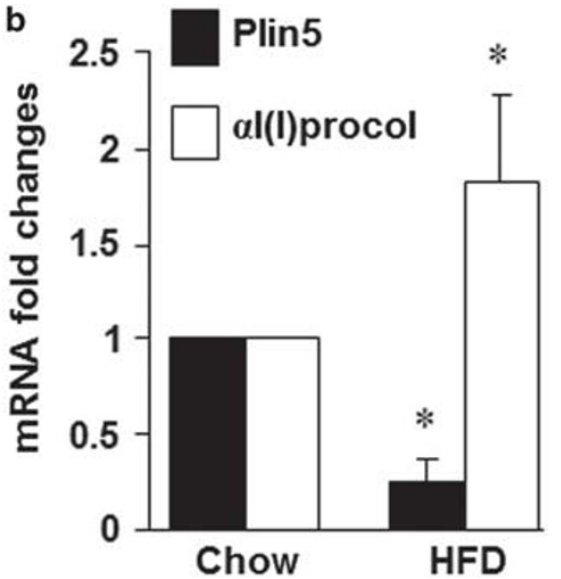

C

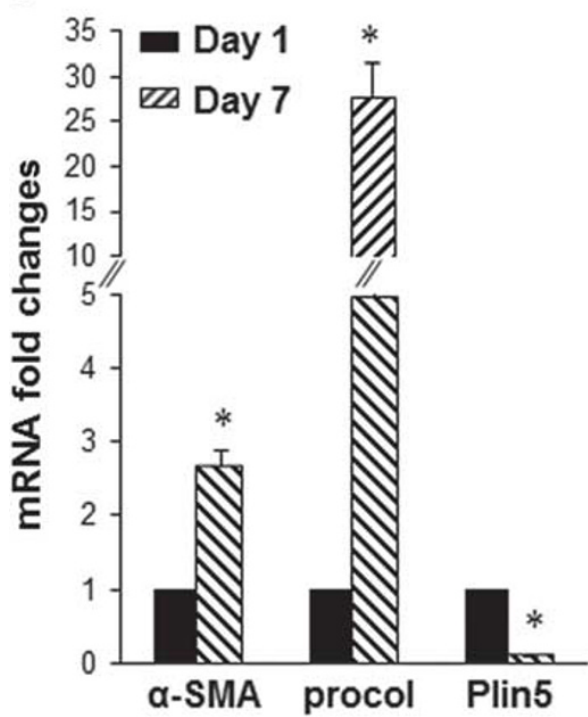

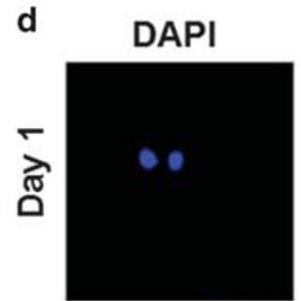

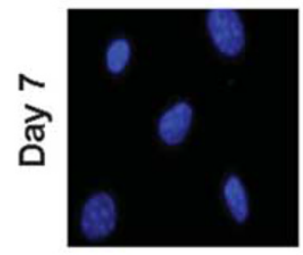

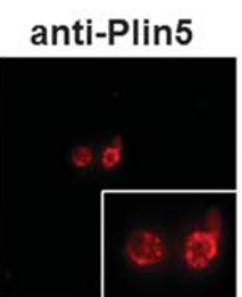

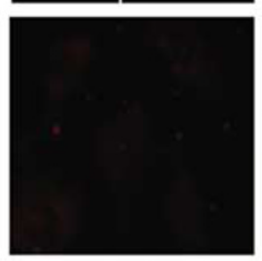

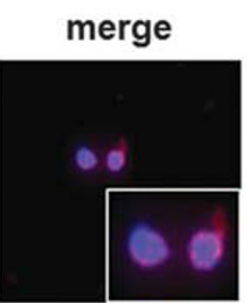

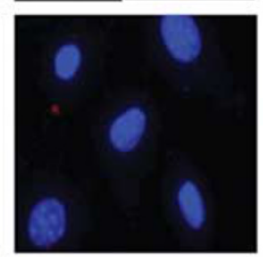

Neg Ctr
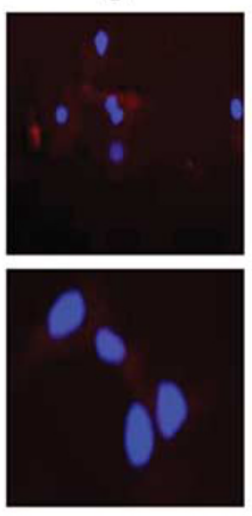

Figure 1 Plin5 is highly expressed in quiescent HSC and dramatically reduced in HSC on activation in vitro and in vivo. (a,b) Samples from an obese and fibrotic mouse model fed a regular chow diet (Chow) or a HFD. (a). Liver sections were stained with hematoxylin and eosin (left panels), Sirius Red (Central panels), or immuno-stain of $a$-SMA (right panels). Representative views were presented (b). Real-time PCR assays ( $n=3$ ). ${ }^{*} P<0.05$ vs HSC from the chow group. (c,d) Freshly isolated HSC from normal mice were cultured for 1 day (Day 1$)$ or 7 days (Day 7$)$. (c) Real-time PCR assays ( $n=3$ ). ${ }^{*} P<0.05$ vs HSC at Day 1. (d) Immuno-fluorescent stain of Plin5. Nuclei were stained by DAPI. The negative control (Neg ctr) was conducted without the primary anti-Plin5 antibodies. The inserted squares denoted the enlarged cells in the views. Representative views were presented.

in media with palmitic acids (PA) at $20 \mu \mathrm{M}$ for $24 \mathrm{~h}$, HSC were fixed and stained with Oil Red O. As shown in Figure 3a, compared with the mock HSC (left panel), or HSC with LV-YFP (middle panel), the expression of exogenous Plin5 apparently restored the formation of LDs in HSC (right panel). PA here was to provide a source of FFA, which had no direct impact on the formation of LDs in mock HSC or LV-YFP (Figure 3a).

To localize the functional domain of Plin5 for the LD formation, recombinant lentiviruses with different fragments of Plin5 cDNA were created, which expressed truncated domains of Plin5. Passaged HSC were respectively transducted with these lentiviruses. After selection with puromycin, cells were cultured in media with PA at $20 \mu \mathrm{M}$ for $24 \mathrm{~h}$. LDs in the cells were stained with Oil Red O. As shown in
Figure $3 \mathrm{~b}$, the major functional domain of Plin5 for the LD formation in HSC resided in the domain of amino acids 1-188 (aa1-188) of Plin5. Splitting this domain into aa1-116 and aa117-188 dramatically eliminated its function of the LD formation, if any left in aa1-116. Expression of a large portion of Plin5 (aa189-463) showed no function in the LD formation.

To clarify the impact of Plin 5 on cellular lipid content in HSC, the foresaid HSC were used for analyzing cellular lipid content. As shown in Figures $3 c$ and $d$, the expression of exogenous Plin5 dramatically elevated the levels of cellular FFA and TGs in HSC. These results collectively supported our postulation that expression of Plin5 restored the formation of LDs and elevated cellular lipid content in HSC, likely leading to the inhibition of HSC activation. 


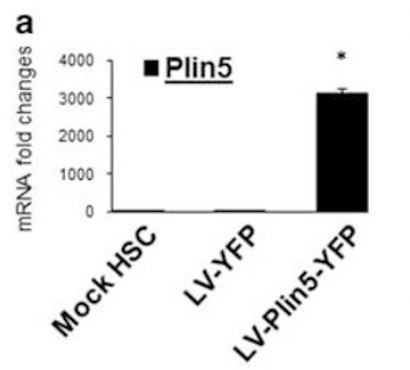

b
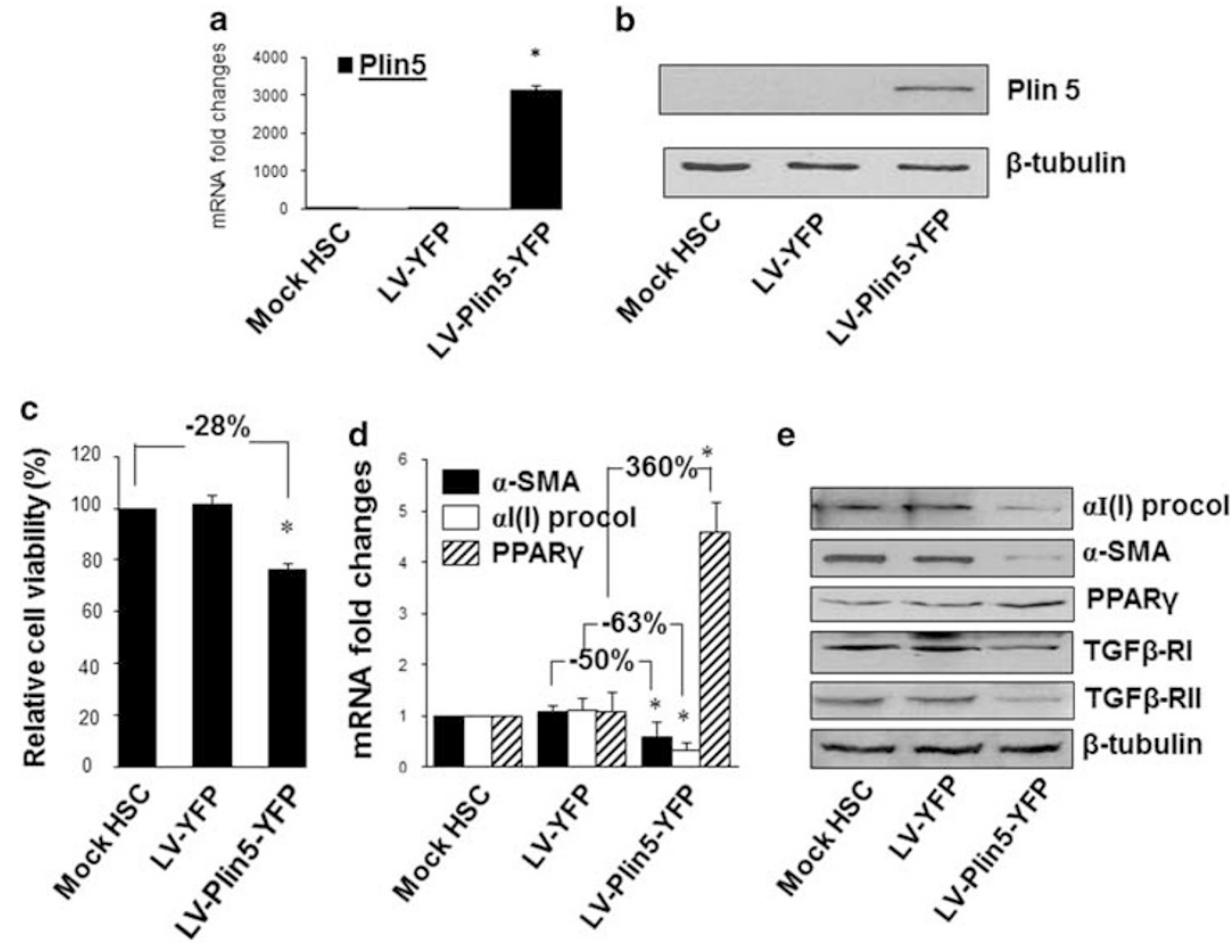

Figure 2 The expression of exogenous Plin5 inhibits HSC activation. Culture-activated HSC (4-9 passages in culture) were transfected with the recombinant lentiviral LV-Plin5-YFP, or the vehicle lentiviral LV-YFP, or with no transduction (Mock HSC). ${ }^{*} P<0.05$ vs mock HSC. Representatives were presented from three independent western blotting analyses. $\beta$-tubulin was used as an invariant control for equal loading. (a) Real-time PCR assays $(n=3)$; (b) western blotting analyses; (c) cell proliferation assays (ie, MTS assays) $(n=3)$; (d) real-time PCR assays $(n=3)$; (e) western blotting analyses.

\section{The Expression of Exogenous Plin5 Regulates Major Modulators for Lipid Homeostasis and Expression of Genes Related with Lipid Metabolism in HSC}

To explore the mechanisms by which the expression of exogenous Plin5 elevated cellular lipid content, effects of plin5 expression on major lipid metabolic modulators and genes were studied. Passaged HSC were transducted with LV-Plin5YFP or LV-YFP. After selection with puromycin, these cells were respectively transfected with the plasmid PPRE-Luc, LXR-Luc, or Topflash. PPRE-Luc was utilized for analyzing the transcriptional activity of $\operatorname{PPAR} \gamma$, whereas LXR-Luc was used for evaluating the transcriptional activity of LXR. Topflash was a luciferase activity reporter plasmid used for assessing the activation of the $\mathrm{Wnt} / \beta$-catenin signaling pathway. As shown in Figure 4a by luciferase activity assays, compared with LV-YFP, LV-Plin5-YFP significantly increased luciferase activities in HSC with LXR-Luc or PPRE-Luc, meanwhile, dramatically reduced the luciferase activity in HSC with Topflash. These results indicated that the expression of exogenous Plin5 significantly stimulated transcriptional activities of LXR and PPAR $\gamma$, and apparently interfered with the $\mathrm{Wnt} / \beta$-catenin signaling pathway in HSC.

Both PPAR $\gamma$ and LXR are the master moderators of adipogenic genes. ${ }^{38,39}$ It was, thus, plausible to further elucidate impacts of Plin5 on expression of lipid metabolic genes in HSC. Passaged HSC were transducted with
LV-Plin5-YFP, or LV-YFP, or no transduction. After selection with puromycin and culture in media with PA at $20 \mu \mathrm{M}$ for $24 \mathrm{~h}$, total RNA from the cells was prepared for real-time PCR. As shown in Figures 4b-d, compared with HSC with no transduction (Mock HSC) or with LV-YFP, the expression of exogenous Plin5 in HSC with LV-Plin5-YFP significantly increased mRNA levels of the major pro-lipogenic regulator sterol receptor element binding protein-1 (SREBP-1) (Figure 4b) and pro-fatty acid synthetic genes, including acetyl coA carboxylase (ACC), fatty acid synthase (FAS), and PPAR $\gamma$ co-activator-1 $\alpha$ (PGC-1 $\alpha$ ) (Figure 4c). Interestingly, LV-Plin5-YFP significantly reduced mRNA levels of pro-fatty acid beta-oxidation genes, including $\operatorname{PPAR} \alpha$ and liver type carnitine palmitoyl transferase (LCPT), and pro-lipolytic hormone-sensitive lipase (HSL) (Figure 4d). Taken together, these data indicated that expression of Plin5-stimulated expression or activity of pro-lipogenic genes and inhibited expression of pro-lipolytic genes in HSC, leading to the elevation of cellular lipid content in HSC.

The Expression of Exogenous Plin5 Dramatically Reduces the Level of ATGL and abhd5 in HSC

Plin5 has been reported to regulate hydrolysis of TG via interacting with adipose triglyceride lipase (ATGL), the rate-limiting lipase, and $\alpha$ - $\beta$-hydrolase domain-containing 5 (abhd5). ${ }^{40-43}$ For fully activation, ATGL must be associated 
a

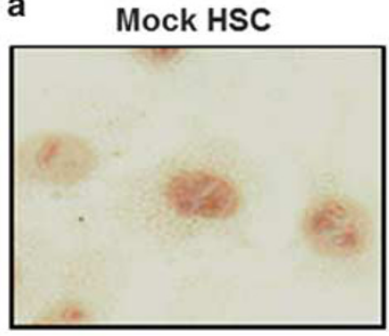

LV-YFP

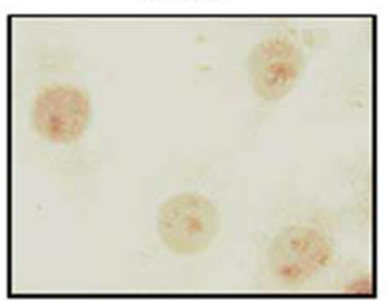

LV-Plin5-YFP

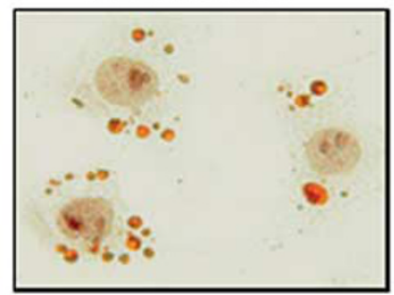

b

Full size aa1-463

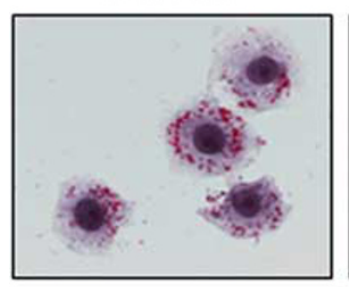

c

c

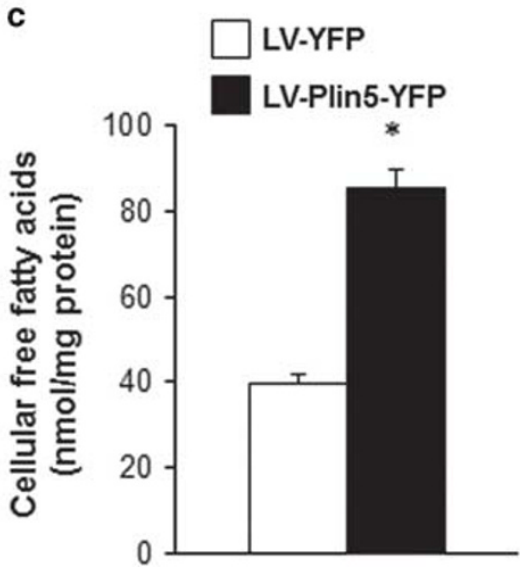

aa1-188
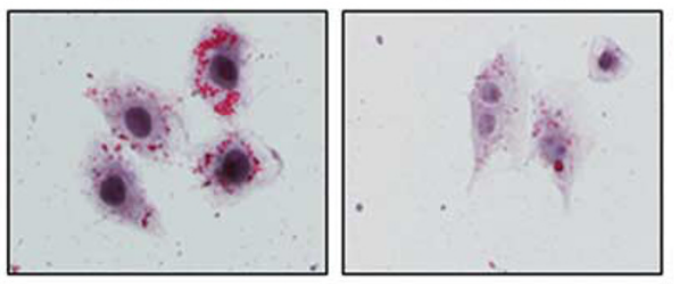

aa117-188

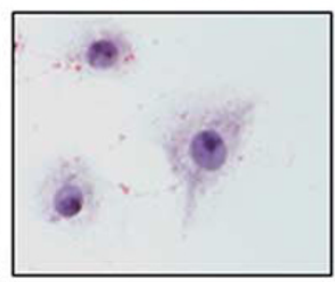

d $\square$ LV-YFP

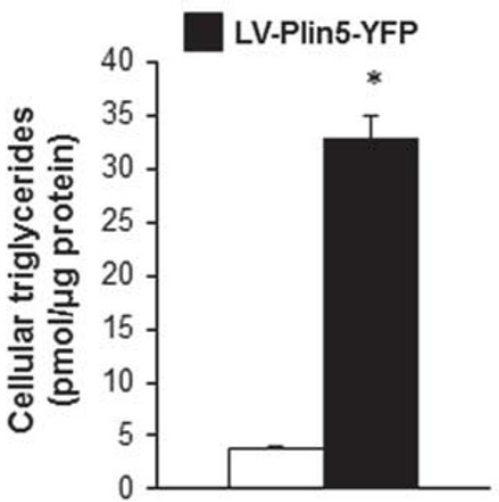

Figure 3 Exogenous Plin5 restores the formation of LD and elevates cellular lipid content in activated HSC. Passaged HSC were transducted with LVPlin5-YFP, or LV-YFP, or no transduction (Mock HSC) $(\mathbf{a}, \mathbf{c}, \mathbf{d})$, or transducted with recombinant lentiviruses with different fragments of Plin5 cDNA, which expressed truncated domains of Plin5 (b). After selection with puromycin, cells were cultured in media with PA at $20 \mu \mathrm{M}$ for $24 \mathrm{~h}$. (a,b) After fixation, LDs in HSC were stained with Oil Red O. Representative views were presented. (c) Analyses of cellular FFA $(n=3), * P<0.05$ vs mock HSC. (d) Analyses of cellular TG $(n=3) .{ }^{*} P<0.05$ vs mock HSC.

with the activator abhd5. ${ }^{44}$ It is of interest to explore the role of Plin5 in regulating expression of ATGL and abhd5 in HSC. Passaged HSC were transducted with LV-Plin5-YFP, or LV-YFP, or no transduction. After selection with puromycin, total RNA and whole-cell extracts from the cells were prepared for real-time PCR and western blotting analyses. As shown in Figure 5, compared with HSC with no transduction, or with LV-YFP, the expression of exogenous Plin5 in HSC with LV-Plin5-YFP significantly suppressed gene expression of ATGL and abhd5 at levels of transcript (Figure 5a) and protein (Figure 5b). These data indicated that the expression of exogenous Plin5 reduced the interaction of ATGL and abhd5 by suppressing their expression, which implied suppression of hydrolysis of lipids and facilitation of the lipid accumulation in HSC.
The Expression of Exogenous Plin5 Stimulates the Activation of AMPK in HSC, Which, in Turn, Induces the Expression of Endogenous Plin5 and is Required for the Plin5-Restored Formation Cellular LDs in HSC and the Inhibition of HSC Activation

To elucidate the mechanisms by which the expression of Plin 5 resulted in the regulation of expression of genes involved in lipid metabolisms, we presumed that the expression of exogenous Plin5 stimulated the activation of AMPK in HSC, which, in turn, induced expression of endogenous Plin5, leading to the restoration of LD formation and the regulation of genes involved in lipid metabolisms and the elevation of cellular lipid content. To study our presumption, passaged HSC were transducted with LV-Plin5-YFP, or LV-YFP, or no transduction. As shown in Figure 6a by 


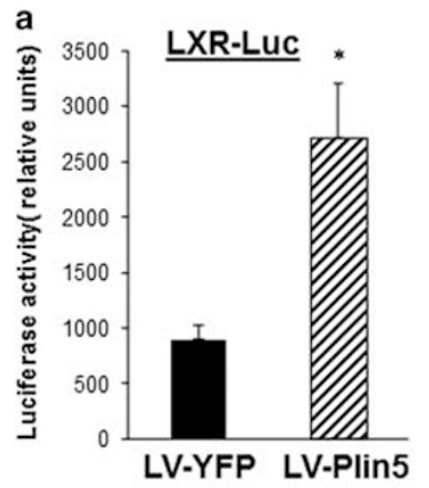

b

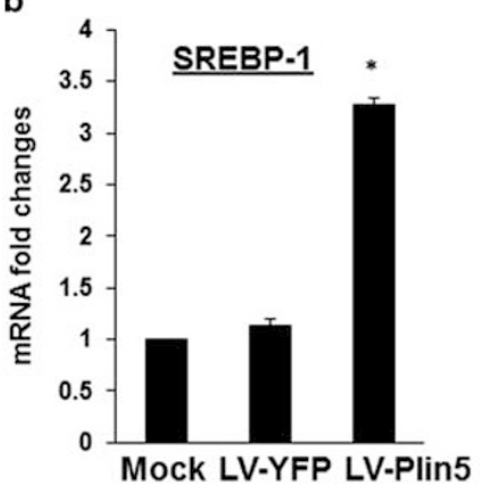

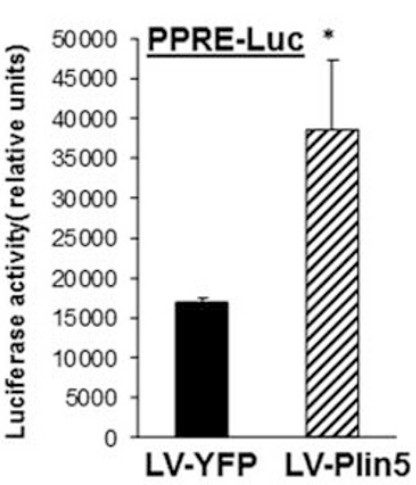

c

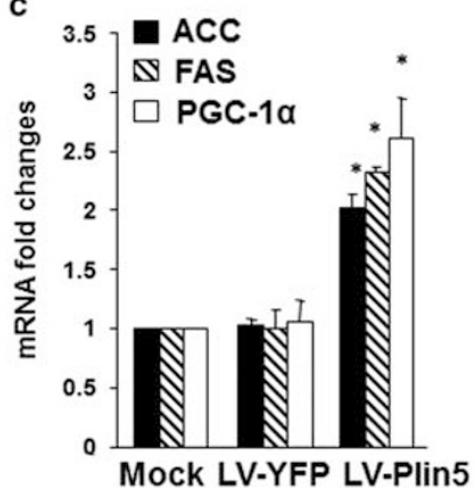

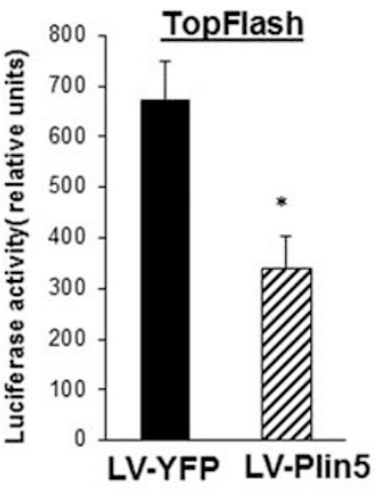

d

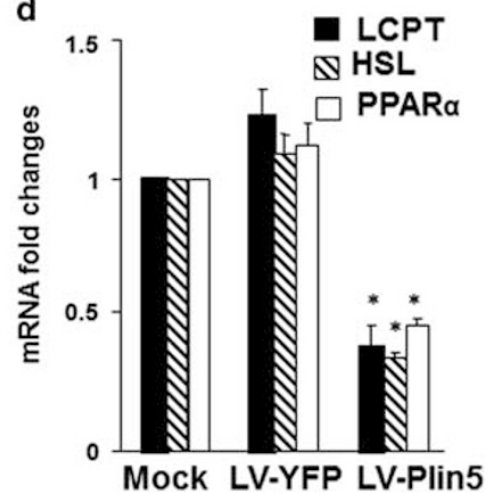

Figure 4 The expression of exogenous Plin5 regulates major modulators for lipid homeostasis and expression of genes related with lipid metabolism in HSC. (a) Passaged HSC were transducted with LV-Plin5-YFP (LV-Plin5) or LV-YFP. After selection with puromycin, these cells were respectively transfected with the plasmid PPRE-Luc, LXR-Luc, or Topflash. Luciferase activity assays were conducted $(n=6) .{ }^{*} P<0.05$ vs HSC transducted with LV-YFP.

(b-d) Passaged HSC were transducted with LV-Plin5-YFP (LV-Plin5), or LV-YFP, or no transduction (Mock HSC). After selection of positively transducted cells with puromycin, total RNA was prepared for real-time PCR assays $(n=3) .{ }^{*} P<0.05$ vs mock HSC.
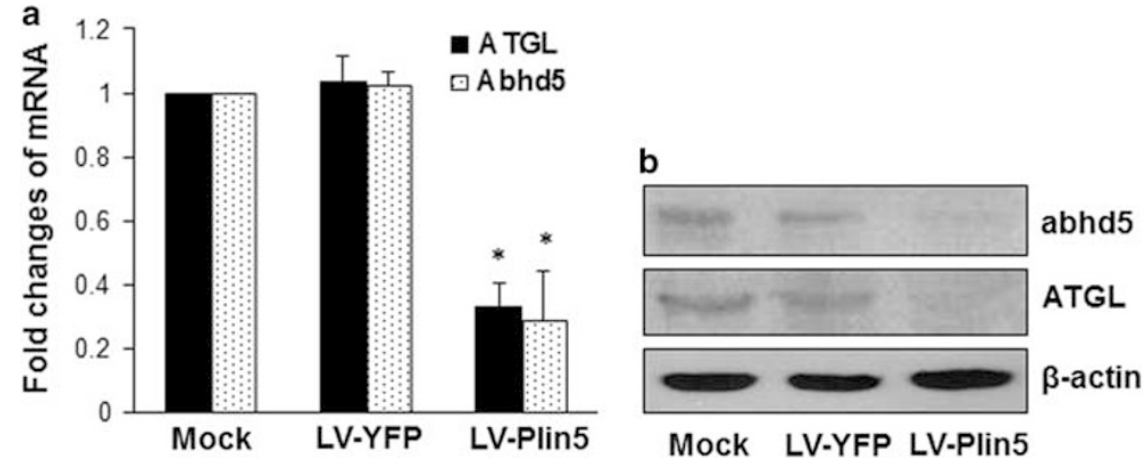

Figure 5 The expression of exogenous Plin 5 dramatically reduces the level of ATGL and abhd5 in HSC. Passaged cells were transducted with LV-Plin5YFP (LV-Plin5), or LV-YFP, or no transduction. After selection, total RNA and whole-cell extracts from the cells were prepared for real-time PCR assays (a) and western blotting analyses $(\mathbf{b}) .{ }^{*} P<0.05$ vs mock HSC $(n=3)$. Representatives were presented from three independent western blotting analyses. $\beta$-actin was used as an invariant control for equal loading.

western blotting analyses, compared with HSC with no transduction (Mock) or with LV-YFP, the expression of exogenous Plin5 in HSC with LV-Plin5-YFP apparently increased the abundance of phosphorylated AMPK, which indicated the activation of AMPK. ${ }^{45}$ In contrast, total AMPK was not changed. Further experiments indicated that inhibition of AMPK activation by the specific AMPK inhibitor Compd $\mathrm{C}$ at various concentrations dose-dependently eliminated the role of Plin5 in the restoration of $\mathrm{LD}$ formation in HSC (Figure 6b).

To determine if the activation of AMPK could induce expression of endogenous Plin5, HSC were transiently 
transfected with the luciferase activity reporter plasmid pPlin5-Luc, which contained a $5^{\prime}$ flanking fragment of mouse Plin5 promoter cDNA in pGL3-Basic. After transfection, cells were treated with AICAR, an AMPK activator, or the AMPK inhibitor Compd C, at various concentrations for $24 \mathrm{~h}$.
Luciferase activity assays indicated that the activation of AMPK by AICAR caused a dose-dependent increase in luciferase activity (Figure 6c). In contrast, the inhibition of AMPK by Compd $\mathrm{C}$ resulted in a dose-dependent reduction in luciferase activity (Figure 6d). These results suggested that a

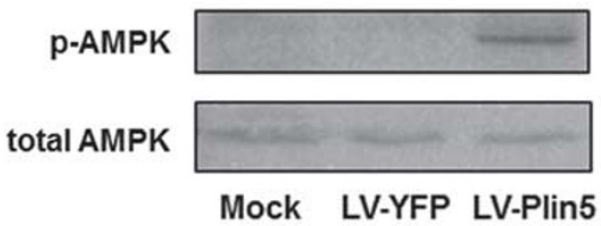

C

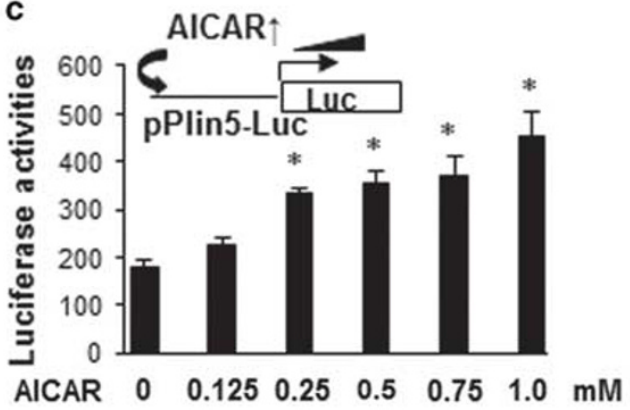

e
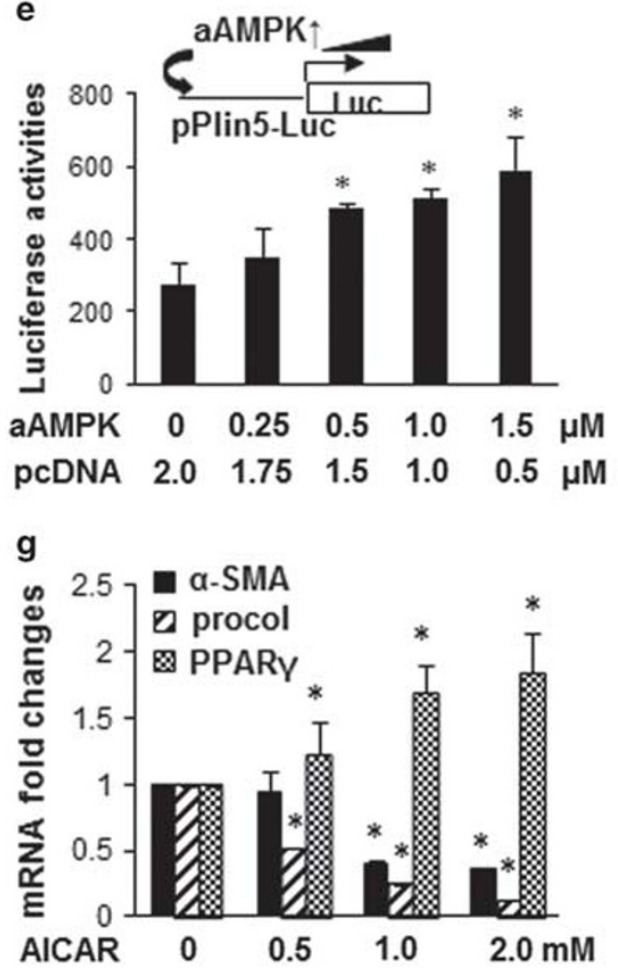

b
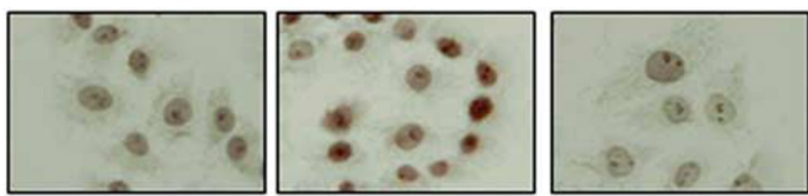

LV-YFP
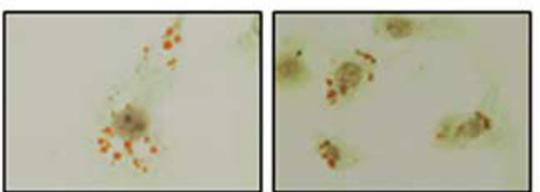

2

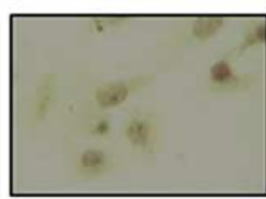

$20 \mu \mathrm{M}$

\section{Compd C}

d

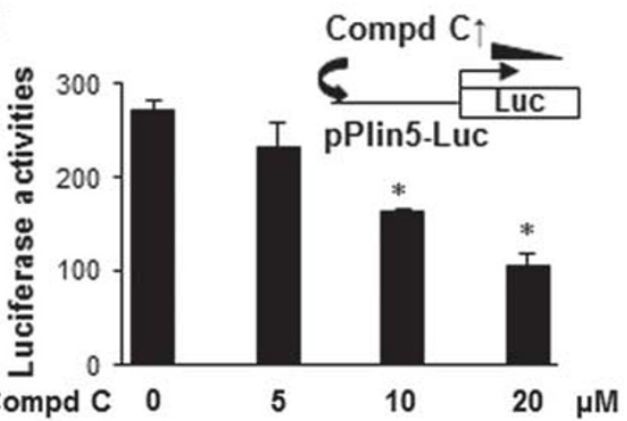

f

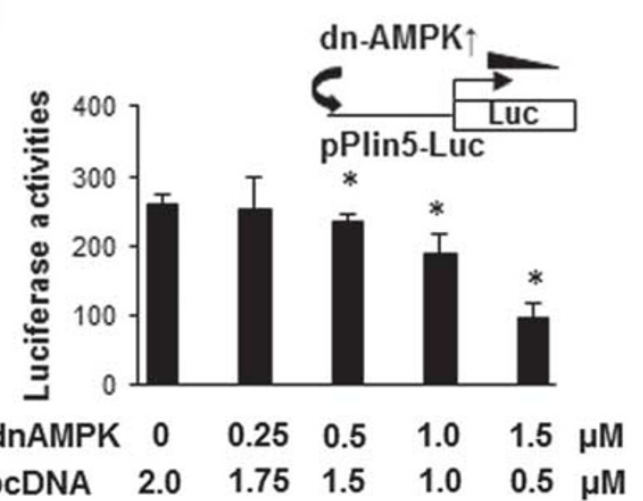

h

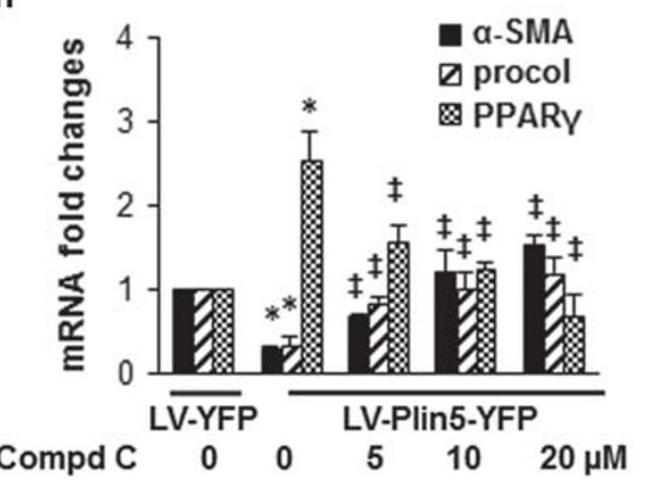


the activation of AMPK could induce expression of endogenous Plin5 in HSC. To further validate the role of the activation of AMPK in regulating gene expression of Plin5, HSC were co-transfected with pPlin5-Luc and paAMPK, or pdn-AMPK. paAMPK expressed constitutively activated AMPK, whereas pdn-AMPK expressed dominantnegative AMPK. ${ }^{33}$ Luciferase activity assays indicated that aAMPK caused a dose-dependent increase in luciferase activity (Figure 6e). However, dn-AMPK resulted in a dosedependent reduction in luciferase activity (Figure 6f). Taken together, these results indicated that the expression of exogenous Plin5 stimulated the activation of AMPK in HSC, which, in turn, induced endogenous plin5 expression. The AMPK activation was required for the Plin5-restored formation cellular LDs in HSC.

Further experiments were conducted to clarify if the activation of AMPK could inhibit HSC activation. Passaged HSC were treated with the AMPK activator AICAR at indicated concentrations for $24 \mathrm{~h}$. Real-time PCR assays indicated that the activation of AMPK by AICAR caused a dose-dependent reduction in mRNA levels of $\alpha \mathrm{I}(\mathrm{I})$ procollagen and $\alpha$-SMA in HSC and an elevation of mRNA level of PPAR $\gamma$ (Figure 6g). In addition, passaged HSC were transducted with LV-Plin5-YFP, or LV-YFP as a control. After selection by puromycin, the cells were treated with the selective AMPK inhibitor Compd C at indicated concentrations for $24 \mathrm{~h}$. Real-time PCR assays were conducted. As shown in Figure 6h, compared with the control lentivirus LV-YFP, LV-Plin5-YFP, as expected, significantly reduced mRNA levels of $\alpha \mathrm{I}(\mathrm{I})$ procollagen and $\alpha$-SMA and increased the level of PPAR $\gamma$ mRNA in HSC. As we showed earlier, expression of exogenous Plin5-activated AMPK (Figure 6a). This function of exogenous Plin5 was eliminated by the AMPK inhibitor Compd $\mathrm{C}$ in a dose-dependent manner (Figure $6 \mathrm{~h}$ ). These results indicated that the activation of AMPK by Plin5 played a critical role in the inhibition of HSC activation.

\section{The Activity of AMPK Regulates Cellular Lipid Content in $\mathrm{HSC}$}

To explore the role of AMPK in regulating cellular lipid content in HSC, passaged HSC were transducted with LV-Plin5-YFP or LV-YFP. After selection with puromycin, HSC were treated with the AMPK inhibitor Compd C, or the AMPK activator AICAR, at various concentrations for $24 \mathrm{~h}$. Cellular lipid content, including FFA and TG, were analyzed. As shown in Figures $7 \mathrm{a}$ and b, compared with LV-YFP, the expression of exogenous Plin5 by LV-Plin5-YFP, as expected, dramatically elevated the levels of cellular FFA (Figure 7a) and TG (Figure 7b) (the corresponding left first columns). However, the inhibition of AMPK by Compd C dose-dependently eliminated the role of Plin5 in elevating cellular FFA (Figure 7a) and TG (Figure 7b) in HSC.

Additional experiments in Figures $7 \mathrm{c}$ and $d$ indicated that further stimulation of the AMPK activation by AICAR caused a dose-dependent, but mild, increase in the level of cellular FFA (Figure 7c) and TG (Figure 7d) in HSC. These results indicated that the activity of AMPK regulated cellular lipid content in HSC.

\section{The Expression of Exogenous Plin5 Attenuates Cellular Oxidative Stress in HSC}

We showed in Figure 2 that the expression of exogenous Plin5 inhibited HSC activation. We presumed that the inhibition of HSC activation by Plin5 was, at least partially, caused by attenuating oxidative stress. To test the presumption, passaged HSC were transducted with or without LV-Plin5-YFP or LV-YFP. Cellular oxidative stress in these cells were analyzed by measuring levels of cellular ROS, lipid peroxides (LPO), GSH, the most abundant thiol antioxidant in mammalian cells, and the ratio of GSH/GSSG, the index of cellular oxidative stress. As shown in Figure 8, compared with HSC with no transduction (Mock) or with LV-YFP, the expression of exogenous Plin5 in HSC with LV-Plin5-YFP significantly increased the content of cellular

\footnotetext{
Figure 6 The expression of exogenous Plin5 stimulates the activation of AMPK in HSC, which, in turn, induces the expression of endogenous Plin5 and is required for the Plin5-restored formation cellular LDs in HSC. (a) Passaged HSC were transducted with LV-Plin5-YFP (LV-Plin5), LV-YFP, or no transduction. After selection with puromycin, cell extracts were prepared for western blotting analyses. Representatives were presented from three independent experiments. Total AMPK was used as an invariant control for equal loading. (b) Passaged HSC were transducted with LV-Plin5-YFP (LVPlin5) or LV-YFP. After selection with puromycin, cells were treated with Compd C at indicated doses for 24 h. LDs in the cells were stained with Oil Red O. Representative views were presented. (c,d) Passaged HSC were transiently transfected with the plasmid pPlin5-Luc. After transfection, cells were treated with the AMPK activator AICAR (c), or the specific AMPK inhibitor Compd C (d), at indicated concentrations for 24 h. Luciferase activity assays were conducted $(n=6)$. ${ }^{*} P<0.05$ vs cells without treatment. The floating schema denoted the plasmid pPlin5-Luc in use for transfection and the application of AICAR or Compd C to the system. (e,f) Passaged HSC in 6-well plates were co-transfected with a total of $4.5 \mu \mathrm{g}$ of a DNA mixture per well, including $2 \mu \mathrm{g}$ of pPlin5-Luc, $0.5 \mu \mathrm{g}$ of pSV- $\beta$-gal, and $2 \mu \mathrm{g}$ of the cDNA expression plasmid paAMPK (e), or pdn-AMPK (f), at indicated doses plus the empty vector pcDNA. The latter was used to ensure an equal amount of total DNA in transfection assays. Luciferase activity assays were conducted $(n=6)$. ${ }^{*} P<0.05$ vs cells transfected with pcDNA at $2 \mu \mathrm{g}$ (the corresponding left first column). The floating schema denoted the plasmid pPlin5-Luc in use for co-transfection with paAMPK or pdn-AMPK. (g,h) Passaged HSC were transducted with LV-Plin5-YFP or LV-YFP (h), or no transduction (g). Cells were treated with AICAR $(\mathbf{g})$ or Compd C (h) at indicated concentrations for $24 \mathrm{~h}$. mRNA levels of $a \mathrm{l}(\mathrm{I})$ procollagen (procol), $a$-SMA, and PPAR $y$ were analyzed by real-time PCR $(n=3)$. ${ }^{*} P<0.05$ vs cells transducted with LV-YFP $(\mathbf{h})$, or no treatment $(\mathbf{g}) ; \neq P<0.05$ vs cells transducted with LV-Plin5-YFP without treatment.
} 

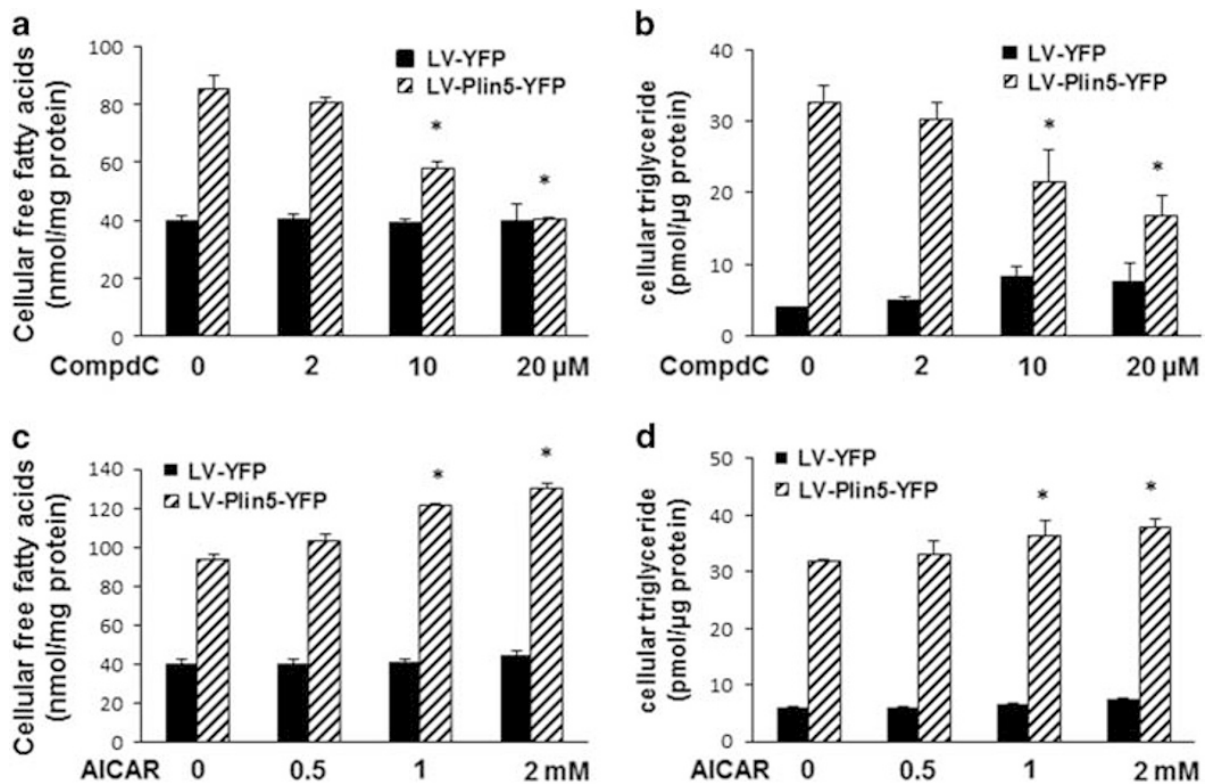

Figure 7 The activity of AMPK regulates cellular lipid content in HSC. Passaged HSC were transducted with LV-Plin5-YFP or LV-YFP. After selection with puromycin, HSC were treated with the AMPK inhibitor Compd C $(\mathbf{a}, \mathbf{b})$, or the AMPK activator AICAR $(\mathbf{c}, \mathbf{d})$, at indicated concentrations for $24 \mathrm{~h} .{ }^{*} P<0.05$ vs cells with LV-Plin5-YFP, but with no treatment $(n=3)$ (the corresponding left second column). (a,c) Cellular FFA assays; (b,d) cellular TG assays.

a
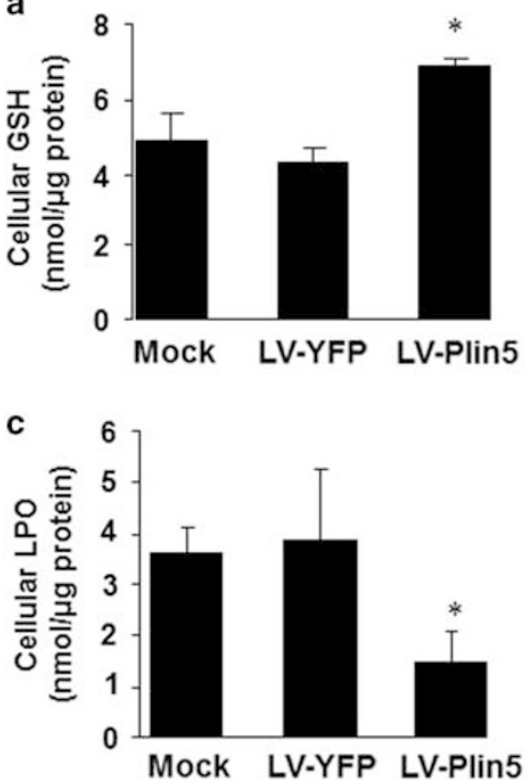

b
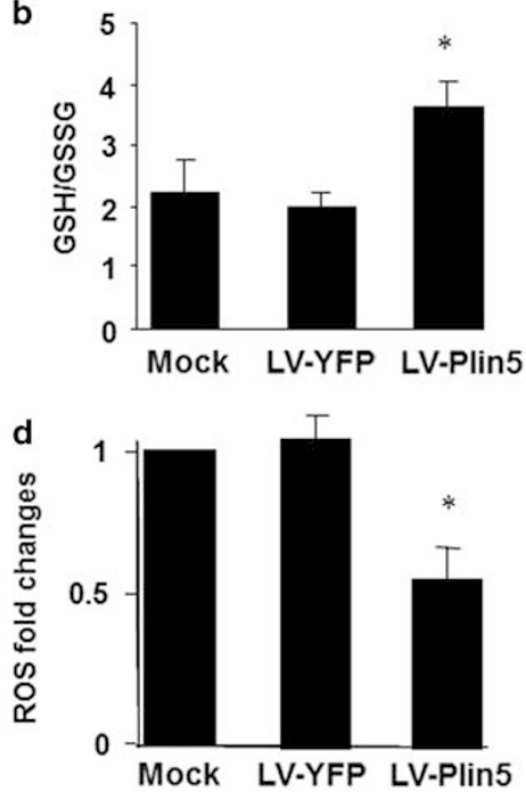

Figure 8 The expression of exogenous Plin5 attenuates cellular oxidative stress in HSC. Passaged HSC were transducted with LV-Plin5-YFP (LV-Plin5), or LV-YFP, or no transduction (Mock). After selection with puromycin, cellular oxidative stress in these cells were analyzed $(n=3)$ ) ${ }^{*} P<0.05$ vs mock cells. (a) Cellular GSH assays; (b) analyses of the ratio of GSH to GSSG; (c) cellular LPO assays; (d) cellular ROS assays.

GSH (Figure 7a) and the ratio of GSH to GSSG (Figure 7b) and dramatically reduced the levels of cellular LPO (Figure 7c) and ROS (Figure 7d). These results demonstrated that the expression of exogenous Plin5 in HSC attenuated cellular oxidative stress.
The Expression of Exogenous Plin5 Induces Gene Expression of GCL Subunits in HSC, Likely by Stimulating the Transcriptional Activity of Nrf2

To explore underlying mechanisms by which expression of Plin5 attenuated oxidative stress in HSC, we postulated that 
a

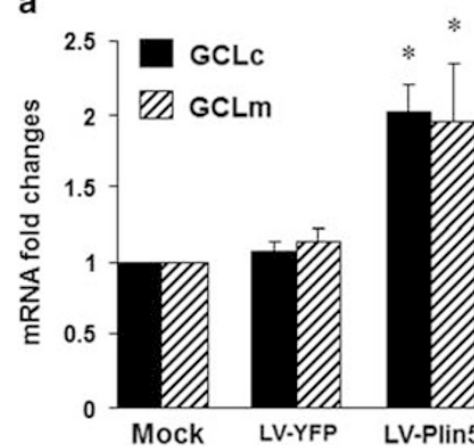

b

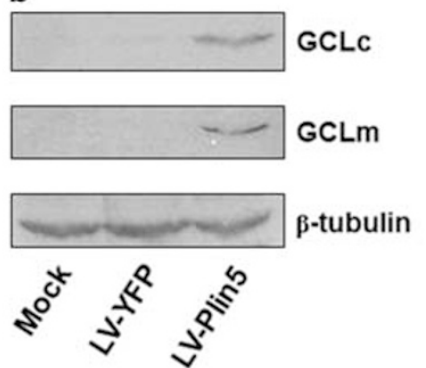

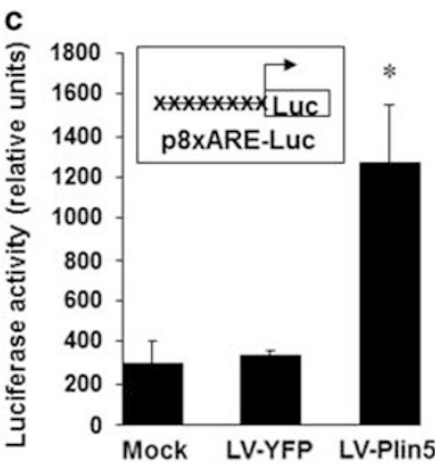

Figure 9 The expression of exogenous Plin5 induces gene expression of GCL subunits in HSC, likely by stimulating the transcriptional activity of Nrf2. Passaged HSC were transducted with LV-Plin5-YFP (LV-Plin5), or LV-YFP, or no transduction (Mock). After selection with puromycin, some of the cells were transfected with the plasmid p8xARE-Luc for luciferase assays. Cells were cultured for additional $24 \mathrm{~h}$. (a). Real-time PCR assays. *P<0.05 vs mock cells $(n=3)$; (b) western blotting analyses. Representatives were presented from three independent experiments. $\beta$-tubulin was used as an invariant control for equal loading; (c) luciferase activity assays. ${ }^{*} P<0.05$ vs mock cells $(n=6)$. The floating schema denoted the plasmid p8x-ARE-Luc in use for transfection to the system.

expression of Plin5-induced gene expression of GCL subunits in HSC, which resulted in de novo synthesis of GSH. To test the postulation, passaged HSC were transducted with LV-Plin5-YFP, or LV-YFP, or no transduction. Total RNA and whole-cell extracts were prepared respectively for realtime PCR and western blotting analyses. As shown in Figure 9, compared with HSC with no transduction (Mock) or with LV-YFP, the expression of exogenous Plin5 in HSC with LV-Plin5-YFP significantly increased the abundance of GCLc and GCLm at both levels of mRNA (Figure 9a) and protein (Figure 9b).

To further elucidate the mechanisms of Plin5 in inducing gene expression of GCL subunits, HSC were transfected with the plasmid p8xARE-Luc, which contained eight copies of the transcription factor Nrf2 binding sites in the promoter. Luciferase activity assays in Figure $9 \mathrm{c}$ indicated that compared with HSC with no transduction (Mock) or with LV-YFP, the expression of exogenous Plin5 in HSC with LV-Plin5-YFP significantly elevated the luciferase activity in these cells, suggesting that Plin5 dramatically induced the transcriptional activity of the transcription factor Nrf2. Taken together, these results indicated that the expression of exogenous Plin5-induced gene expression of GCL subunits in HSC likely by stimulating the transcriptional activity of $\mathrm{Nrf} 2$.

\section{DISCUSSION}

Our results in the present report demonstrated that the activation of HSC was coupled to a dramatic reduction in the expression of Plin5 in vitro and in vivo. The expression of exogenous Plin5 significantly elevated the levels of cellular lipid content and restored the formation of LDs in HSC, which were mediated by the activation of AMPK. In addition, the expression of exogenous Plin5 attenuated cellular oxidative stress in HSC. These actions collectively resulted in the inhibition of HSC activation. Our results, for the first time, reported the role of Plin5 in the formation of cellular
LDs in HSC and the impact of its expression on the inhibition of HSC activation. Our results support the proposal of an important role of cellular lipids in maintaining HSC in quiescent state. ${ }^{12,13}$ Restoration of lipogenesis in HSC might be a strategy for attenuating HSC activation and inhibiting fibrogenesis. ${ }^{12,13}$

Quiescent HSC contain large amounts of retinoids in LDs, which is a unique feature of HSC. ${ }^{7,8,11}$ Activation of HSC is coupled with the loss of retinoids and LDs. ${ }^{11}$ Lecithin-retinol acyltransferase acts as the sole retinol acyltransferase in the liver and is responsible for the formation of retinyl esters in HSC. ${ }^{1}$ Lecithin-retinol acyltransferase is highly expressed in HSC and downregulated during HSC activation. ${ }^{46}$ Lrat $^{-/-}$ mice showed a striking total absence of large lipid-containing droplets that normally store hepatic retinoid within HSC. ${ }^{47}$ It is interesting that the absence of retinyl ester-containing LDs does not promote spontaneous, bile duct ligation-induced and carbon tetrachloride-induced liver fibrosis. ${ }^{46}$ Our pilot experiments showed that the expression of exogenous Plin5 had no apparent impact on the expression of endogenous lecithin-retinol acyltransferase in HSC (data not shown here). Additional experiments are necessary to determine if any retinoids exist in the Plin5-induced LDs in HSC. Our prediction is that there is few retinoids, if not any. Further studies are required for evaluating the significance of LDs in the inhibition of HSC activation.

It remains unclear the direct relationship of the cause and consequence between the reduction of cellular lipid content and loss of LD and HSC activation. Our results in this report showed that the restoration of LD formation and elevation of cellular lipid content by expression of exogenous Plin5 significantly inhibited HSC activation in vitro. Although not unequivocal evidence, it strongly suggests the role of LDs and lipid content in maintaining HSC in quiescent state.

Maintenance of lipid homeostasis is crucial to cell functions. In addition to fatty acid uptake/transportation 
cross the cell membrane, cellular lipid content is mainly regulated by a complex process of lipogenesis, fatty acid $\beta$-oxidation, and lipid hydrolysis. Hepatic lipogenesis at transcriptional level is regulated by a group of protein regulators, including the transcription factor SREBP-1c, ${ }^{48}$ as well as the nuclear receptors PPAR $\gamma$, and LXR. ${ }^{38,39}$ The activation of LXR activation stimulates lipogenesis via SREBP-1c. ${ }^{49,50}$ There is a potential interaction or crosstalk between LXR and PPAR $\gamma .{ }^{39}$ We and others have shown that expression of PPAR $\gamma$ and/or other pro-lipogenic genes could reverse activated HSC to differentiated cells. ${ }^{12,13,29} \mathrm{Wnt}$ signaling is implicated in human fibrotic diseases, such as pulmonary fibrosis, renal fibrosis, and liver fibrosis. Blocking the canonical Wnt signal pathway with the co-receptor antagonist Dickkopf-1 restores gene expression of PPAR $\gamma$ and inhibits the activation of HSC. ${ }^{51}$ We observed in this report that the expression of exogenous Plin5 induced the activity of LXR and PPAR $\gamma$, and interrupted the canonical Wnt signal pathway, as well as stimulated the expression of SREBP-1c in HSC. These effects collectively promoted the expression of pro-lipogenic genes and facilitated the elevation of cellular lipid content in HSC.

On the other hand, lipolysis is regulated by three lipases, adipose triglyceride lipase (ATGL), the rate-limiting lipase that initiates lipolysis by hydrolyzing the first ester bond and releasing the first fatty acid; hormone-sensitive lipase (HSL), which has a high affinity for diacylglycerol as a substrate; and, finally, monoacylglycerol lipase. ${ }^{52}$ Lipolysis occurs on the surface of intracellular LDs. For fully activation, ATGL must be associated with the activator abhd5. ${ }^{44}$ Abhd5 in cytosol is directed to LDs by LD proteins. ${ }^{41}$ Interruption of their interactions could greatly affect the activity of ATGL. ${ }^{40}$ Mutations of either abhd5 or ATGL result in ectopic lipid accumulation in skin, muscle, and liver. ${ }^{53}$ At the molecular level, independent work from groups strongly supports the idea that Plin5 plays as a scaffold for three major key lipolytic players: abhd5, ATGL, and HSL in hydrolysis of TG in LDs. ${ }^{40-43}$ It has been shown that the C-terminus of Plin5 serves as an ATGL and abhd5 binding domain. ${ }^{54}$ ATGL and abhd5 competitively bind Plin $5 .{ }^{41}$ The interaction between abhd5 and Plin5 is dynamic and essential for regulating the activity of ATGL at LDs. ${ }^{40}$ Activation of protein kinases has shown its roles in modulating lipid homeostasis. Phosphorylation of Plin5 by protein kinase A fuels lipolysis by stimulating its interaction with ATGL. ${ }^{43}$ On the other hand, AMPK acts as a nutrient sensor ${ }^{55}$ and an anti-lipolytic factor by phosphorylating and inactivating HSL ${ }^{56}$ and blocking its translocation to $\mathrm{LDs}^{57}$ Protein kinase A and AMPK played opposite roles in regulation of lipid content in HSC and in the inhibition of HSC activation. ${ }^{23,58}$ In this study, we observed that the expression of exogenous Plin5 induced the activity of AMPK. The inhibition of AMPK activity by its inhibitor Compd C eliminated the roles of Plin5 in elevating cellular lipids and in restoring the formation of LDs in HSC. Our pilot experiments showed that Plin5 could be phosphorylated by
AMPK in HSC (data not shown). In addition, we showed a direct link between the activation of AMPK and the inhibition of HSC, using the AMPK activation AICAR, and the role of Plin5-caused AMPK activation in the inhibition of HSC activation, using the selective AMPK inhibitor Compd C. Accumulating evidence has indicated that AMPK modulates the proliferation and myofibrogenic activation of HSC via multiple signaling mechanisms. ${ }^{59}$ Recent studies indicated that activation of AMPK markedly attenuated TGF- $\beta 1$ functions, ${ }^{60}$ and interrupted the TGF- $\beta$ signaling pathway by regulating transcriptional coactivator p300 in HSC. ${ }^{61}$ Additional experiments are ongoing to further explore the impact of AMPK-caused phosphorylation of Plin5 on the protein-protein interaction between Plin5 and ATGL or abhd5, and its role in the reduction of lipolysis in HSC.

Oxidative stress plays a critical role in HSC activation and liver fibrosis, regardless of etiology. ${ }^{62,63}$ Anti-oxidant molecule GSH reacts with ROS or functions as a cofactor of antioxidant enzymes, resulting in the protection of functions of redox-sensitive molecules, including enzymes and transcription factors. ${ }^{64}$ Plin5 is highly expressed in oxidative tissues $^{42,65}$ and is involved in the interaction between LDs and mitochondria. ${ }^{54}$ Knockout of Plin5 in mice increases oxidative stress to the heart. ${ }^{20}$ It has been reported that the activation of AMPK promotes the phosphorylation of the transcription factor Nrf2, which triggers the translocation of Nrf2 from the cytoplasm into the nucleus, leading to its binding to ARE and the induction of expression of target genes. ${ }^{66}$ In this report, we demonstrated that the expression of exogenous Plin5 reduced cellular ROS and LPO, and attenuated oxidative stress in HSC by elevating the level of cellular GSH and GSH/GSSG ratio. The effects were likely mediated by activating Nrf2 and inducing the expression of GCL subunits, GCLc and GCLm. Our observations are consistent with prior reports that Nrf2 played a critical role in regulating expression of GCLc and GCLm. ${ }^{67,68}$ Our results suggest that the elevation of intracellular redox status might be one of the mechanisms by which Plin5 inhibits HSC activation.

On the basis of our observations, a simplified model is proposed to explain the inhibitory effects of Plin5 on HSC activation (Figure 10). Expression of Plin5 is depleted in HSC on activation. The expression of exogenous Plin5 in HSC restores the formation of cellular LDs in HSC and stimulates the activation of AMPK, which, in turn, induces the expression of endogenous Plin5. The activation of AMPK results in the elevation of cellular lipid content in HSC by inducing lipogenesis and suppressing lipolysis. On the other hand, the activation of AMPK stimulates the transcription activity of Nrf2 and the expression of GCL subunits, leading to the elevation of cellular levels of GSH and the attenuation of cellular oxidative stress in HSC. These effects collectively result in the inhibition of HSC activation. It bears emphasis that our results do not exclude any other roles or mechanisms of Plin5 in the inhibition of HSC activation. Because our 


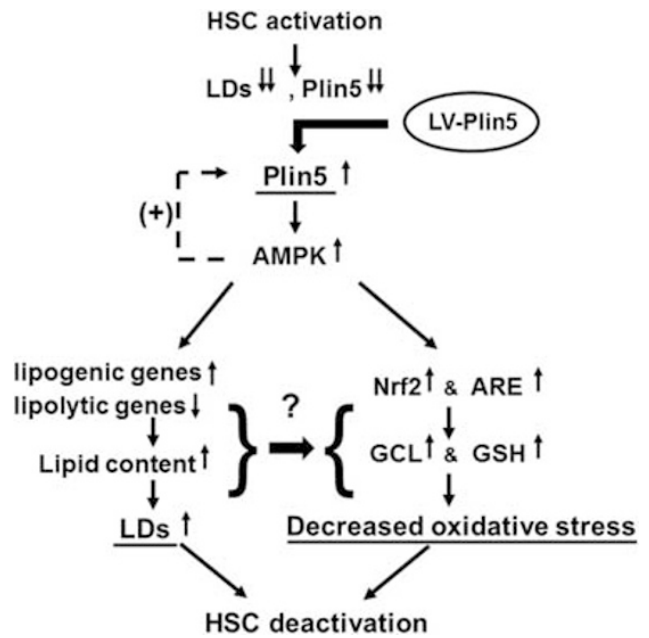

Figure $10 \mathrm{~A}$ simplified action model for Plin5 to inhibit the activation of HSC. Expression of Plin5 is depleted in HSC on activation. Introduction of exogenous Plin5 in HSC by transduction of LV-Plin5-YFP (LV-Plin5) restores the formation of cellular LDs in HSC and stimulates the activation of AMPK, which, in turn, induces the expression of endogenous Plin5. The activation of AMP results in the elevation of cellular lipid content in HSC by inducing lipogenesis and suppressing lipolysis. On the other hand, the activation of AMPK stimulates the transcription activity of Nrf2 and the expression of GCL subunits, leading to the elevation of cellular levels of GSH and the attenuation of cellular oxidative stress in HSC. These effects collectively inhibit HSC activation.

current studies excluded the roles driven by other hepatic cells and factors, it must be very cautious to make any conclusion derived from the in vitro studies.

The protective effects of LD protein Plin5 on the inhibition of HSC activation observed in this report are novel and experiments are ongoing to further explore the underlying mechanisms and clinical applications. As expression of Plin5 is gradually reduced in HSC during liver injury, it is plausible to use Plin5 as a biomarker for determining the activation of HSC and for predicting the progress of hepatic fibrogenesis in patients. In addition, Plin 5 might be a candidate for gene therapy in treatment of liver fibrosis. These potential applications of Plin5 are being explored in our laboratory.

\section{ACKNOWLEDGMENTS}

The work was supported by Doisy Research Fund and the Research Award from the Saint Louis University Liver Center to A. Chen. The authors appreciate technical support from Youcai Tang.

\section{DISCLOSURE/CONFLICT OF INTEREST}

The authors declare no conflict of interest.

1. Blaner WS, O'Byrne SM, Wongsiriroj $\mathrm{N}$ et al. Hepatic stellate cell lipid droplets: a specialized lipid droplet for retinoid storage. Biochim Biophys Acta 2009;1791:467-473.

2. Kimmel AR, Brasaemle DL, McAndrews-Hill $M$ et al. Adoption of PERILIPIN as a unifying nomenclature for the mammalian PAT-family of intracellular lipid storage droplet proteins. J Lipid Res 2010;51:468-471.

3. Paul A, Chan L, Bickel PE. The PAT family of lipid droplet proteins in heart and vascular cells. Curr Hypertens Rep 2008;10:461-466.
4. Yamaguchi T, Matsushita S, Motojima K et al. MLDP, a novel PAT family protein localized to lipid droplets and enriched in the heart, is regulated by peroxisome proliferator-activated receptor alpha. J Biol Chem 2006;281:14232-14240.

5. Okumura T. Role of lipid droplet proteins in liver steatosis. J Physiol Biochem 2011;67:629-636.

6. Wang C, Zhao Y, Gao X et al. Perilipin 5 improves hepatic lipotoxicity by inhibiting lipolysis. Hepatology 2015;61:870-882.

7. Puche JE, Saiman Y, Friedman SL. Hepatic stellate cells and liver fibrosis. Compr Physiol 2013;3:1473-1492.

8. Trautwein C, Friedman SL, Schuppan D et al. Hepatic fibrosis: concept to treatment. J Hepatol 2015;62:S15-S24.

9. Pinzani M. Pathophysiology of non-alcoholic steatohepatitis and basis for treatment. Dig Dis 2011;29:243-248.

10. Wells RG. Liver fibrosis: challenges of the new era. Gastroenterology 2009;136:387-388.

11. Friedman SL. Mechanisms of hepatic fibrogenesis. Gastroenterology 2008;134:1655-1669.

12. Tsukamoto $\mathrm{H}$, She $\mathrm{H}$, Hazra $\mathrm{S}$ et al. Anti-adipogenic regulation underlies hepatic stellate cell transdifferentiation. J Gastroenterol Hepatol 2006;21:S102-S105.

13. Tsukamoto $\mathrm{H}$, She $\mathrm{H}$, Hazra $\mathrm{S}$ et al. Fat paradox of steatohepatitis. J Gastroenterol Hepatol 2008;23:S104-S107.

14. Lee TF, Mak KM, Rackovsky O et al. Downregulation of hepatic stellate cell activation by retinol and palmitate mediated by adipose differentiation-related protein (ADRP). J Cell Physiol 2010;223: 648-657.

15. Fridovich I. The biology of oxygen radicals. Science 1978;201:875-880.

16. Jones DP. Redox potential of GSH/GSSG couple: assay and biological significance. Methods Enzymol 2002;348:93-112.

17. Fraser JA, Kansagra $P$, Kotecki $C$ et al. The modifier subunit of Drosophila glutamate-cysteine ligase regulates catalytic activity by covalent and noncovalent interactions and influences glutathione homeostasis in vivo. J Biol Chem 2003;278:46369-46377.

18. Seelig GF, Simondsen RP, Meister A. Reversible dissociation of gammaglutamylcysteine synthetase into two subunits. J Biol Chem 1984;259: 9345-9347.

19. Sekura R, Meister A. gamma-Glutamylcysteine synthetase. Further purification, "half of the sites" reactivity, subunits, and specificity. J Biol Chem 1977;252:2599-2605.

20. Kuramoto K, Okamura $\mathrm{T}$, Yamaguchi $\mathrm{T}$ et al. Perilipin 5, a lipid droplet-binding protein, protects heart from oxidative burden by sequestering fatty acid from excessive oxidation. J Biol Chem 2012;287: 23852-23863.

21. Fu $\mathrm{Y}$, Zheng $\mathrm{S}$, Lin $\mathrm{J}$ et al. Curcumin protects the rat liver from $\mathrm{CCl} 4$-caused injury and fibrogenesis by attenuating oxidative stress and suppressing inflammation. Mol Pharmacol 2008;73:399-409.

22. Kang $\mathrm{Q}$, Chen A. Curcumin suppresses expression of low-density lipoprotein (LDL) receptor, leading to the inhibition of LDL-induced activation of hepatic stellate cells. Br J Pharmacol 2009;157:1354-1367.

23. Tang $Y$, Chen A. Curcumin prevents leptin raising glucose levels in hepatic stellate cells by blocking translocation of glucose transporter-4 and increasing glucokinase. Br J Pharmacol 2010;161:1137-1149.

24. Tetri LH, Basaranoglu M, Brunt EM et al. Severe NAFLD with hepatic necroinflammatory changes in mice fed trans fats and a high-fructose corn syrup equivalent. Am J Physiol Gastrointest Liver Physiol 2008;295:G987-G995.

25. Chen A, Tang Y, Davis $\vee$ et al. Liver fatty acid binding protein (L-Fabp) modulates murine stellate cell activation and diet-induced nonalcoholic fatty liver disease. Hepatology 2013;57:2202-2212.

26. Lin J, Tang $Y$, Kang $Q$ et al. Curcumin eliminates the inhibitory effect of advanced glycation end-products (AGEs) on gene expression of AGE receptor-1 in hepatic stellate cells in vitro. Lab Invest 2012;92:827-841.

27. Schmittgen TD, Zakrajsek BA, Mills AG et al. Quantitative reverse transcription-polymerase chain reaction to study mRNA decay: comparison of endpoint and real-time methods. Anal Biochem 2000;285:194-204.

28. Lin J, Chen A. Curcumin diminishes the impacts of hyperglycemia on the activation of hepatic stellate cells by suppressing membrane translocation and gene expression of glucose transporter-2. Mol Cell Endocrinol 2011;333:160-171.

29. Zheng S, Chen A. Activation of PPARgamma is required for curcumin to induce apoptosis and to inhibit the expression of extracellular 
matrix genes in hepatic stellate cells in vitro. Biochem J 2004;384: 149-157.

30. Steffensen KR, Holter E, Alikhani N et al. Glucocorticoid response and promoter occupancy of the mouse LXRalpha gene. Biochem Biophys Res Commun 2003;312:716-724.

31. DasGupta R, Kaykas A, Moon RT et al. Functional genomic analysis of the Wnt-wingless signaling pathway. Science 2005;308:826-833.

32. Wang XJ, Hayes JD, Wolf CR. Generation of a stable antioxidant response element-driven reporter gene cell line and its use to show redox-dependent activation of nrf2 by cancer chemotherapeutic agents. Cancer Res 2006;66:10983-10994.

33. Woods A, Azzout-Marniche D, Foretz M et al. Characterization of the role of AMP-activated protein kinase in the regulation of glucoseactivated gene expression using constitutively active and dominant negative forms of the kinase. Mol Cell Biol 2000;20:6704-6711.

34. Feng $Y$, Nie $L$, Thakur MD et al. A multifunctional lentiviral-based gene knockdown with concurrent rescue that controls for off-target effects of RNAi. Genomics Proteomics Bioinformatics 2010;8:238-245.

35. Lin J, Tang $Y$, Kang $Q$ et al. Curcumin inhibits gene expression of receptor for advanced glycation end-products (RAGE) in hepatic stellate cells in vitro by elevating PPARgamma activity and attenuating oxidative stress. Br J Pharmacol 2012;166:2212-2227.

36. Lin J, Zheng S, Chen A. Curcumin attenuates the effects of insulin on stimulating hepatic stellate cell activation by interrupting insulin signaling and attenuating oxidative stress. Lab Invest 2009;89: 1397-1409.

37. Zheng S, Yumei F, Chen A. De novo synthesis of glutathione is a prerequisite for curcumin to inhibit hepatic stellate cell (HSC) activation. Free Radic Biol Med 2007;43:444-453.

38. Savage DB. PPAR gamma as a metabolic regulator: insights from genomics and pharmacology. Expert Rev Mol Med 2005;7:1-16.

39. Parikh M, Patel K, Soni S et al. Liver X receptor: a cardinal target for atherosclerosis and beyond. J Atheroscler Thromb 2014;21:519-531.

40. Granneman JG, Moore HP, Mottillo EP et al. Functional interactions between Mldp (LSDP5) and Abhd5 in the control of intracellular lipid accumulation. J Biol Chem 2009;284:3049-3057.

41. Granneman JG, Moore HP, Mottillo EP et al. Interactions of perilipin-5 (Plin5) with adipose triglyceride lipase. J Biol Chem 2011;286: 5126-5135.

42. Wolins NE, Quaynor BK, Skinner JR et al. OXPAT/PAT-1 is a PPARinduced lipid droplet protein that promotes fatty acid utilization. Diabetes 2006;55:3418-3428.

43. Wang $H$, Bell $M$, Sreenivasan $U$ et al. Unique regulation of adipose triglyceride lipase (ATGL) by perilipin 5, a lipid droplet-associated protein. J Biol Chem 2011;286:15707-15715.

44. Yamaguchi T. Crucial role of CGI-58/alpha/beta hydrolase domaincontaining protein 5 in lipid metabolism. Biol Pharm Bull 2010;33: 342-345.

45. Liu XM, Peyton KJ, Shebib AR et al. Activation of AMPK stimulates heme oxygenase-1 gene expression and human endothelial cell survival. Am J Physiol Heart Circ Physiol 2011;300:H84-H93.

46. Kluwe J, Wongsiriroj N, Troeger JS et al. Absence of hepatic stellate cell retinoid lipid droplets does not enhance hepatic fibrosis but decreases hepatic carcinogenesis. Gut 2011;60:1260-1268.

47. O'Byrne SM, Wongsiriroj N, Libien J et al. Retinoid absorption and storage is impaired in mice lacking lecithin:retinol acyltransferase (LRAT). J Biol Chem 2005;280:35647-35657.
48. Xu X, So JS, Park JG et al. Transcriptional control of hepatic lipid metabolism by SREBP and ChREBP. Semin Liver Dis 2013;33:301-311.

49. Schultz JR, Tu H, Luk A et al. Role of LXRs in control of lipogenesis. Genes Dev 2000;14:2831-2838.

50. Yoshikawa T, Shimano H, Amemiya-Kudo $M$ et al. Identification of liver $X$ receptor-retinoid $X$ receptor as an activator of the sterol regulatory element-binding protein 1c gene promoter. Mol Cell Biol 2001;21: 2991-3000.

51. Miao CG, Yang $Y Y$, He $X$ et al. Wnt signaling in liver fibrosis: progress, challenges and potential directions. Biochimie 2013;95:2326-2335.

52. Thiele C, Spandl J. Cell biology of lipid droplets. Curr Opin Cell Biol 2008;20:378-385.

53. Adachi H, Tsujimoto M. Endothelial scavenger receptors. Prog Lipid Res 2006;45:379-404.

54. Wang H, Sztalryd C. Oxidative tissue: perilipin 5 links storage with the furnace. Trends Endocrinol Metab 2011;22:197-203.

55. Fisslthaler B, Fleming I. Activation and signaling by the AMP-activated protein kinase in endothelial cells. Circ Res 2009;105:114-127.

56. Garton AJ, Yeaman SJ. Identification and role of the basal phosphorylation site on hormone-sensitive lipase. Eur J Biochem 1990;191: 245-250.

57. Daval M, Diot-Dupuy F, Bazin R et al. Anti-lipolytic action of AMPactivated protein kinase in rodent adipocytes. J Biol Chem 2005;280: 25250-25257.

58. Tang Y, Chen A. Curcumin protects hepatic stellate cells against leptininduced activation in vitro by accumulating intracellular lipids. Endocrinology 2010;151:4168-4177.

59. Ceni E, Mello T, Galli A. Pathogenesis of alcoholic liver disease: role of oxidative metabolism. World J Gastroenterol 2014;20: 17756-17772.

60. Thakur S, Viswanadhapalli S, Kopp JB et al. Activation of AMP-activated protein kinase prevents TGF-beta1-induced epithelial-mesenchymal transition and myofibroblast activation. Am J Pathol 2015;185: 2168-2180.

61. Lim JY, Oh MA, Kim WH et al. AMP-activated protein kinase inhibits TGF-beta-induced fibrogenic responses of hepatic stellate cells by targeting transcriptional coactivator p300. J Cell Physiol 2012;227: 1081-1089.

62. De Minicis S, Brenner DA. Oxidative stress in alcoholic liver disease: role of NADPH oxidase complex. J Gastroenterol Hepatol 2008;23: S98-103.

63. Di Sario A, Candelaresi C, Omenetti A et al. Vitamin E in chronic liver diseases and liver fibrosis. Vitam Horm 2007;76:551-573.

64. Wu G, Fang $Y Z$, Yang $S$ et al. Glutathione metabolism and its implications for health. J Nutr 2004;134:489-492.

65. Dalen KT, Dahl T, Holter E et al. LSDP5 is a PAT protein specifically expressed in fatty acid oxidizing tissues. Biochim Biophys Acta 2007;1771:210-227.

66. Kobayashi M, Yamamoto M. Molecular mechanisms activating the Nrf2-Keap1 pathway of antioxidant gene regulation. Antioxid Redox Signal 2005;7:385-394.

67. Zhang $\mathrm{H}$, Court N, Forman HJ. Submicromolar concentrations of 4-hydroxynonenal induce glutamate cysteine ligase expression in HBE1 cells. Redox Rep 2007;12:101-106.

68. Wild AC, Moinova HR, Mulcahy RT. Regulation of gammaglutamylcysteine synthetase subunit gene expression by the transcription factor Nrf2. J Biol Chem 1999;274:33627-33636. 